\title{
An Eighteen-Node Hybrid-Stress Solid-Shell Element for Homogenous and Laminated Structures
}

\author{
K.Y.Sze, L.-Q.Yao \\ Departmentsof Mechanical Engineering, The University of Hong Kong \\ Pokfulam Road, Hong Kong, P.R.CHINA
}

and

\section{T.H.H.Pian}

Department of Aeronautics \& Astronautics, Massachusetts Institute of Technology

Cambridge, MA 02139, U.S.A.

\begin{abstract}
Solid-shell elements are often equipped with two layers of nodes. Thus, the thickness (normal) strain along the thickness direction is essentially constant. When these elements are subjected to pure bending, the shrinkage/expansion induced by the in-plane strain and the Poisson's ratio coupling in the upper and lower halves of the elements cancel each other. With a constant thickness strain, the plane strain state is resulted that leads to thickness locking. In this paper, a modified generalized laminate stiffness matrix is devised to resolve not only the thickness locking but also some abnormalities of solid-shell elements in laminate analyses. Associated with the modified matrix, a set of generalized stresses can be defined and a modified Hellinger-Reissner functional can be derived by treating the generalized stresses as the independent variables. Based on the functional, an eigenteen-node hybrid-stress solid-shell element suitable for laminate analyses is proposed via a stabilization approach. All the benchmark tests indicate that the present stabilized element is close to the reduced integration element in accuracy.
\end{abstract}

Keywords : Solid shell, hybrid stress, finite element, composite, laminates, stabilization 


\section{INTRODUCTION}

Solid-Shell elements which possess no rotational d.o.f.s and are applicable to thin plate/shell analyses have attracted considerable attention [1-9]. Compared to the degenerated-shell elements, solid-shell elements are advantageous in the following aspects. Firstly, solid-shell elements are simpler in their geometric and kinetic descriptions. Secondly, no special effort is required for matching the translations in solid elements and rotations in shell elements when a structure is composed of solid and thin-walled regions. The laborious task of defining algebraic constraints or introducing solid-to-shell transition elements can be exempted. Thirdly, the complication on handling finite rotations can be avoided. Nevertheless, formulating robust solid-shell elements is indeed more demanding than formulating robust degenerated-shell elements. While the latter elements are only bothered by shear and membrane lockings, the former elements can also be plagued by thickness and trapezoidal lockings $[10,11]$.

To resolve thickness locking in homogeneous elements, enhanced assumed strain modes [5,6], hybrid-stress formulation $[3,9,12]$ and the plane stress enforcement $[1,2,4,7,8]$ have been resorted to. Among them, only hybrid-stress formulation and the plane stress enforcement remain effective to secure the plane stress condition in laminate plates/shells. Unfortunately, the conventional hybridstress method is not suitable for formulating laminated degenerated-shell and solid-shell formulations due to the zig-zag nature of the stresses which cannot be easily accounted for in the assumed stress shape functions. On the other hand, the plane stress enforcement gives rise to incorrect thickness strain and sharp discontinuities in the thickness stress when the elements are sandwiched.

To be computationally efficient, most solid-shell elements are formed by only two layers of nodes. Hence, the thickness strain is essentially constant along the thickness direction and can only represent the thickness average of its pointwise value. In this paper, a constitutive relation is derived for the bending strain, membrane strain, average thickness strain, bending stress, membrane stress and the thickness stress by assuming that the thickness stress is independent of the transverse coordinate. The assumption is indeed adopted in some hybrid-stress elements that overcome thickness locking $[3,9,12]$. It will be demonstrated that the relation can resolve some inconsistent predictions associated with the enhanced assumed strain method and the plane stress enforcement.

As mentioned, conventional hybrid-stress method is not suitable for formulating laminated degenerated- and solid-shell elements. Hence, hybrid-strain or assumed-strain elements dominate the laminated shell analysis [2,13] though hybrid-stress elements are generally more accurate and efficient, e.g. see [14]. By employing the proposed constitutive relation, a hybrid variational 
functional can be derived by introducing a set of generalized stresses as independent variables. Using the functional, a stabilized hybrid-stress eighteen-node solid-shell element previously derived by Sze, Yi \& Tay [7] is extended to laminate analyses. The salient feature of the element is that the element stiffness can be partitioned into a lower and a higher order matrices. While the former is equivalent to the reduced integrated element, the latter plays the role of stabilizing the former. The stabilized element is computationally efficient as the higher order matrix can be formed in the element subroutine without resorting to numerical integration.

\section{THE CONVENTIONAL EIGHTEEN-NODE SOLID-SHELL ELEMENT}

In this section, formulation of the conventional eighteen-node solid-shell element will be briefed. Without losing generality, the natural coordinate $\zeta \in[-1,+1]$ is always aligned with the transverse direction of the shell as shown in Figure 1. For the element, the geometric and displacement can be obtained from standard interpolation as :

$$
\begin{aligned}
& \mathbf{X}(\xi, \eta, \zeta)=\sum_{i=1}^{9} N_{i}(\xi, \eta) \cdot\left(\frac{1+\zeta}{2} \mathbf{X}_{i}+\frac{1-\zeta}{2} \mathbf{X}_{9+i}\right)=\mathbb{N}(\xi, \eta) \cdot \mathbf{X}_{o}+\zeta \mathbb{N}(\xi, \eta) \cdot \mathbf{X}_{n} \\
& \mathbf{U}(\xi, \eta, \zeta)=\sum_{i=1}^{9} N_{i}(\xi, \eta) \cdot\left(\frac{1+\zeta}{2} \mathbf{U}_{i}+\frac{1-\zeta}{2} \mathbf{U}_{9+i}\right)=\mathbb{N}(\xi, \eta) \cdot \mathbf{q}_{o}+\zeta \mathbb{N}(\xi, \eta) \cdot \mathbf{q}_{n}
\end{aligned}
$$

where

$N_{i}$ 's are the two-dimensional nine-node Lagrangian interpolation functions,

$\mathbf{X}_{i}$ and $\mathbf{U}_{i}$ are respectively the coordinate and displacement vectors of the i-th node,

$\mathbb{N}(\xi, \eta)=\left[N_{1} \mathbf{I}_{3}, \cdots, N_{9} \mathbf{I}_{3}\right], \mathbf{I}_{m}$ denotes the $m$-th order identity matrix,

$$
\mathbf{X}_{o}=\frac{1}{2}\left\{\begin{array}{c}
\mathbf{X}_{1}+\mathbf{X}_{10} \\
\vdots \\
\mathbf{X}_{9}+\mathbf{X}_{18}
\end{array}\right\}, \mathbf{X}_{n}=\frac{1}{2}\left\{\begin{array}{c}
\mathbf{X}_{1}-\mathbf{X}_{10} \\
\vdots \\
\mathbf{X}_{9}-\mathbf{X}_{18}
\end{array}\right\}, \mathbf{q}_{o}=\frac{1}{2}\left\{\begin{array}{c}
\mathbf{U}_{1}+\mathbf{U}_{10} \\
\vdots \\
\mathbf{U}_{9}+\mathbf{U}_{18}
\end{array}\right\}, \mathbf{q}_{n}=\frac{1}{2}\left\{\begin{array}{c}
\mathbf{U}_{1}-\mathbf{U}_{10} \\
\vdots \\
\mathbf{U}_{9}-\mathbf{U}_{18}
\end{array}\right\}
$$

The infinitesimal covariant element strains with respect to the parametric coordinates are :

$$
\begin{aligned}
& \varepsilon_{\xi}=\mathbf{X}_{\xi}^{T} \mathbf{U}_{,_{\xi}}=\varepsilon_{\xi m}+\zeta \varepsilon_{\xi b}+\zeta^{2} \varepsilon_{\xi h}, \varepsilon_{\eta}=\mathbf{X}_{,_{\eta}}^{T} \mathbf{U}_{,_{\eta}}=\varepsilon_{\eta m}+\zeta \varepsilon_{\eta b}+\zeta^{2} \varepsilon_{\eta h}, \\
& 2 \varepsilon_{\xi \eta}=\mathbf{X},_{\xi}^{T} \mathbf{U}_{\eta}+\mathbf{X}_{\eta}^{T} \mathbf{U}_{\xi}=2 \varepsilon_{\xi \eta m}+2 \zeta \varepsilon_{\xi \eta b}+2 \zeta^{2} \varepsilon_{\xi \eta h}, \varepsilon_{\zeta}=\mathbf{X}_{\zeta}^{T} \mathbf{U}_{\zeta}=\mathbf{X}_{n}^{T} \mathbb{N}^{T} \mathbb{N}_{\mathbf{q}_{n}}, \\
& \gamma_{\zeta \xi}=\mathbf{X}_{\zeta}^{T} \mathbf{U}_{,_{\xi}}+\mathbf{X}_{\xi}^{T} \mathbf{U}_{\zeta}=\gamma_{\zeta \xi o}+\zeta \gamma_{\zeta \xi n}, \gamma_{\zeta \eta}=\mathbf{X}_{\zeta}^{T} \mathbf{U}_{\eta}+\mathbf{X}_{,_{\eta}}^{T} \mathbf{U}_{\zeta}=\gamma_{\zeta \eta o}+\zeta \gamma_{\zeta \eta n}
\end{aligned}
$$

where

$$
\varepsilon_{\xi m}=\mathbf{X}_{o}^{T} \mathbb{N}_{\xi \xi}^{T} \mathbb{N}_{\xi} \mathbf{q}_{o}, \varepsilon_{\xi b}=\mathbf{X}_{n}^{T} \mathbb{N}_{\xi}^{T} \mathbb{N}_{\xi} \mathbf{q}_{o}+\mathbf{X}_{o}^{T} \mathbb{N}_{\xi}^{T} \mathbb{N}_{\xi} \mathbf{q}_{n}, \varepsilon_{\xi h}=\mathbf{X}_{n}^{T} \mathbb{N}_{\xi}^{T} \mathbb{N}_{\xi} \mathbf{q}_{n}
$$




$$
\begin{aligned}
& \varepsilon_{\eta m}=\mathbf{X}_{o}^{T} \mathbb{N}_{\eta_{\eta}}^{T} \mathbb{N},_{\eta} \mathbf{q}_{o}, \varepsilon_{\eta b}=\mathbf{X}_{n}^{T} \mathbb{N}_{\eta_{\eta}}^{T} \mathbb{N}_{\jmath_{\eta}} \mathbf{q}_{o}+\mathbf{X}_{0}^{T} \mathbb{N}_{\eta_{\eta}^{T}}^{T} \mathbb{N}_{\eta} \mathbf{q}_{n}, \varepsilon_{\eta h}=\mathbf{X}_{n}^{T} \mathbb{N}_{\eta_{\eta}}^{T} \mathbb{N}_{\eta} \mathbf{q}_{n}, \\
& 2 \varepsilon_{\xi \eta m}=\mathbf{X}_{o}^{T}\left(\mathbb{N}_{\xi \xi}^{T} \mathbb{N}_{\eta}+\mathbb{N}_{\eta}^{T} \mathbb{N}_{\xi \xi}\right) \mathbf{q}_{o}, 2 \varepsilon_{\xi \eta h}=\mathbf{X}_{n}^{T}\left(\mathbb{N}_{, \xi}^{T} \mathbb{N}_{\eta}+\mathbb{N},{ }_{\eta}^{T} \mathbb{N}_{\xi}\right) \mathbf{q}_{n},
\end{aligned}
$$

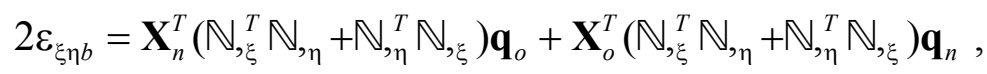

$$
\begin{aligned}
& \gamma_{\zeta \xi o}=\mathbf{X}_{n}^{T} \mathbb{N}^{T} \mathbb{N}, \mathbf{q}_{o}+\mathbf{X}_{o}^{T} \mathbb{N}_{\xi \xi}^{T} \mathbb{N} \mathbf{q}_{n}, \gamma_{\zeta \xi n}=\mathbf{X}_{n}^{T}\left(\mathbb{N}^{T} \mathbb{N},{ }_{\xi}+\mathbb{N}_{, \xi}^{T} \mathbb{N}\right) \mathbf{q}_{n}, \\
& \gamma_{\zeta \eta o}=\mathbf{X}_{n}^{T} \mathbb{N}^{T} \mathbb{N},_{\eta} \mathbf{q}_{o}+\mathbf{X}_{o}^{T} \mathbb{N}_{\eta_{\eta}}^{T} \mathbb{N} \mathbf{q}_{n}, \gamma_{\zeta \eta n}=\mathbf{X}_{n}^{T}\left(\mathbb{N}^{T} \mathbb{N},_{\eta}+\mathbb{N},{ }_{\eta}^{T} \mathbb{N}\right) \mathbf{q}_{n}
\end{aligned}
$$

As the material properties of shells are often defined in a local orthogonal frame $x-y-z$, it is necessary to obtain the local physical strains from the covariant ones. It will be assumed as usual that the z-axis and the $x$-y-plane are parallel to the $\zeta$-axis and mid-surface of the shell, respectively. Hence, the relations between the covariant strains and the local physical strains when approximated by the ones evaluated at the mid-surface are :

$$
\begin{aligned}
& \boldsymbol{\epsilon}_{=}=\left\{\begin{array}{c}
\varepsilon_{x} \\
\varepsilon_{y} \\
2 \varepsilon_{x y}
\end{array}\right\}=\left[\begin{array}{ccc}
x_{\xi}^{2} & x_{\eta}^{2} & 2 x_{\xi} x_{\eta} \\
y_{\xi}^{2} & y_{\eta}^{2} & 2 y_{\xi} y_{\eta} \\
x_{\xi} y_{\xi} & x_{\eta} y_{\eta} & x_{\xi} y_{\eta}+x_{\eta} y_{\xi}
\end{array}\right]^{-T}\left\{\begin{array}{c}
\varepsilon_{\xi} \\
\varepsilon_{\eta} \\
2 \varepsilon_{\xi \eta}
\end{array}\right\}=\mathbf{T}_{\varepsilon}^{-T}\left\{\begin{array}{c}
\varepsilon_{\xi} \\
\varepsilon_{\eta} \\
2 \varepsilon_{\xi \eta}
\end{array}\right\}, \\
& \boldsymbol{\gamma}=\left\{\begin{array}{l}
\gamma_{z x} \\
\gamma_{z y}
\end{array}\right\}=\frac{1}{z_{\zeta}}\left[\begin{array}{cc}
x_{\xi} & x_{\eta} \\
y_{\xi} & y_{\eta}
\end{array}\right]^{-T}\left\{\begin{array}{l}
\gamma_{\zeta \xi} \\
\gamma_{\zeta \eta}
\end{array}\right\}=\mathbf{T}_{\gamma}^{-T}\left\{\begin{array}{l}
\gamma_{\zeta \xi} \\
\gamma_{\zeta \eta}
\end{array}\right\}, \varepsilon_{\|}=\varepsilon_{z}=\frac{1}{z_{\zeta}^{2}} \varepsilon_{\zeta}=\frac{1}{\left\|\mathbf{X}_{n}\right\|^{2}} \varepsilon_{\zeta}
\end{aligned}
$$

where

$$
\begin{aligned}
& x_{\xi}=\mathbf{e}_{x}^{T} \mathbb{N}, \mathbf{X}_{o}, y_{\xi}=\mathbf{e}_{y}^{T} \mathbb{N}_{\xi} \mathbf{X}_{o}, x_{\eta}=\mathbf{e}_{x}^{T} \mathbb{N}, \mathbf{X}_{o}, y_{\eta}=\mathbf{e}_{y}^{T} \mathbb{N}, \mathbf{X}_{o}, z_{\zeta}=\mathbf{e}_{z}^{T} \mathbb{N} \mathbf{X}_{n}, \\
& \mathbf{e}_{x}, \mathbf{e}_{y} \text { and } \mathbf{e}_{z} \text { are the unit vectors along the local x-, y- and z-axes. }
\end{aligned}
$$

By consolidating (3) and (4), the local Cartesian strain can be expressed as :

$$
\begin{aligned}
& \boldsymbol{\epsilon}_{=}=\mathbf{T}_{\varepsilon}^{-T}\left(\left\{\begin{array}{c}
\varepsilon_{\xi m} \\
\varepsilon_{\eta m} \\
2 \varepsilon_{\xi \eta m}
\end{array}\right\}+\zeta\left\{\begin{array}{c}
\varepsilon_{\xi b} \\
\varepsilon_{\eta b} \\
2 \varepsilon_{\xi \eta b}
\end{array}\right\}+\zeta^{2}\left\{\begin{array}{c}
\varepsilon_{\xi h} \\
\varepsilon_{\eta h} \\
2 \varepsilon_{\xi \eta h}
\end{array}\right\}\right)=\boldsymbol{\epsilon}_{m}+\zeta \boldsymbol{\epsilon}_{b}+\zeta^{2} \boldsymbol{\epsilon}_{h}=\left(\mathbb{B}_{m}+\zeta \mathbb{B}_{b}+\zeta^{2} \mathbb{B}_{h}\right) \mathbf{q}, \\
& \boldsymbol{\varepsilon}_{\|}=\mathbb{B}_{\|} \mathbf{q}, \boldsymbol{\gamma}=\mathbf{T}_{\gamma}^{-T}\left(\left\{\begin{array}{c}
\gamma_{\zeta \xi o} \\
\gamma_{\zeta \eta o}
\end{array}\right\}+\zeta\left\{\begin{array}{c}
\gamma_{\zeta \xi n} \\
\gamma_{\zeta \eta n}
\end{array}\right\}\right) \mathbf{q}=\boldsymbol{\gamma}_{o}+\zeta \boldsymbol{\gamma}_{n}=\left(\mathbb{B}_{o}+\zeta \mathbb{B}_{n}\right) \mathbf{q} .
\end{aligned}
$$

where $\mathbf{q}=\left\{\mathbf{U}_{1}^{T}, \cdots, \mathbf{U}_{18}^{T}\right\}^{T}, \boldsymbol{\epsilon}$ 's, $\boldsymbol{\gamma}$ 's and $\mathbb{B}$ 's are self-defined. Moreover, $\mathbb{B}$ 's are independent of $\zeta$. With $z$ being one of the principal material directions, the constitutive relation can be written as :

$$
\left\{\begin{array}{c}
\boldsymbol{\sigma}_{=} \\
\hdashline \sigma_{\|}
\end{array}\right\}=\left[\begin{array}{c:c}
\mathbf{C}_{=} & \mathbf{C}_{\times} \\
\hdashline \mathbf{C}_{\times}^{T} & C_{\|}
\end{array}\right]\left\{\begin{array}{c}
\boldsymbol{\epsilon}_{=} \\
\hdashline \varepsilon_{\|}
\end{array}\right\} \text {or }\left\{\begin{array}{l}
\boldsymbol{\epsilon}_{=} \\
\hdashline \varepsilon_{\|}
\end{array}\right\}=\left[\begin{array}{c:c}
\mathbf{S}_{=} & \mathbf{S}_{\times} \\
\hdashline \mathbf{S}_{\times}^{T} & S_{\|}
\end{array}\right]\left\{\begin{array}{c}
\boldsymbol{\sigma}_{=} \\
\hdashline \sigma_{\|}
\end{array}\right\}, \boldsymbol{\tau}=\left\{\begin{array}{l}
\tau_{z x} \\
\tau_{z y}
\end{array}\right\}=\mathbf{C}_{t}\left\{\begin{array}{l}
\gamma_{z x} \\
\gamma_{z y}
\end{array}\right\}=\mathbf{C}_{t} \boldsymbol{\gamma}
$$

where 


$$
\boldsymbol{\sigma}_{=}=\left\{\sigma_{x}, \sigma_{y}, \sigma_{x y}\right\}^{T}, \boldsymbol{\epsilon}_{=}=\left\{\varepsilon_{x}, \varepsilon_{y}, 2 \varepsilon_{x y}\right\}^{T} \text { and }\left[\begin{array}{c:c}
\mathbf{S}_{=} & \mathbf{S}_{\times} \\
\hdashline \mathbf{S}_{\times}^{T} & S_{\|}
\end{array}\right]=\left[\begin{array}{c:c}
\mathbf{C}_{=} & \mathbf{C}_{\times} \\
\hdashline \mathbf{C}_{\times}^{T} & C_{\|}
\end{array}\right]^{-1}
$$

The element stiffness matrix can be formulated via the elementwise potential energy functional :

$$
\begin{aligned}
\Pi_{P}^{e} & =\frac{1}{2} \int_{V^{e}}\left[\left\{\begin{array}{l}
\boldsymbol{\epsilon}_{=} \\
\varepsilon_{\|}
\end{array}\right\}^{T}\left[\begin{array}{c:c}
\mathbf{C}_{=} & \mathbf{C}_{\times} \\
\hdashline \mathbf{C}_{\times}^{T} & C_{\|}
\end{array}\right]\left\{\begin{array}{l}
\boldsymbol{\epsilon}_{=} \\
\varepsilon_{\|}
\end{array}\right\}+\boldsymbol{\gamma}^{T} \mathbf{C}_{t} \boldsymbol{\gamma}^{T}\right] \mathrm{d} v-P^{e} \\
& \left.=\frac{1}{2} \int_{-1}^{+1+1+1} \int_{-1-1}^{+1}\left[\begin{array}{l}
\boldsymbol{\epsilon}_{=} \\
\varepsilon_{\|}
\end{array}\right\}^{T}\left[\begin{array}{l:l}
\mathbf{C}_{=} & \mathbf{C}_{\times} \\
\hdashline \mathbf{C}_{\times}^{T} & C_{\|}
\end{array}\right]\left\{\begin{array}{l}
\boldsymbol{\epsilon}_{=} \\
\varepsilon_{\|}
\end{array}\right\}+\boldsymbol{\gamma}^{T} \mathbf{C}_{t} \boldsymbol{\gamma}\right] J d \xi d \eta d \zeta-P^{e}
\end{aligned}
$$

where $V^{e}$ is the element domain, $P^{e}$ denotes the elementwise load potential and $J$ is the Jacobian determinant. In formulating the element stiffness matrix, the second order $\zeta$-terms in $\boldsymbol{\epsilon}_{=}$and all the $\zeta$-terms in the Jacobian determinant (a quadratic function of $\zeta$ ) are often truncated. We have compared elements with and without the $\zeta$-terms in Section 7 by a number of singly- and doublycurved shell problems. The noted differences are negligible. For higher computational efficiency, the truncation is adopted and (7) can be simplified as :

$$
\begin{aligned}
\Pi_{P}^{e} & \left.=\frac{1}{2} \int_{-1}^{+1} \int_{-1-1}^{+1+1} \int_{-1}^{+1}\left[\begin{array}{c}
\boldsymbol{\epsilon}_{m}+\zeta \boldsymbol{\epsilon}_{b} \\
\varepsilon_{\|}
\end{array}\right\}^{T}\left[\begin{array}{c:c}
\mathbf{C}_{=} & \mathbf{C}_{\times} \\
\hdashline \mathbf{C}_{\times}^{T} & C_{\|}
\end{array}\right]\left\{\begin{array}{c}
\boldsymbol{\epsilon}_{m}+\zeta \epsilon_{b} \\
\varepsilon_{\|}
\end{array}\right\}+\left(\boldsymbol{\gamma}_{o}+\zeta \boldsymbol{\gamma}_{n}\right)^{T} \mathbf{C}_{t}\left(\boldsymbol{\gamma}_{o}+\zeta \boldsymbol{\gamma}_{n}\right)\right] J_{o} d \xi d \eta d \zeta-P^{e} \\
& =\frac{1}{2}\left\langle\left\{\begin{array}{l}
\boldsymbol{\epsilon}_{m} \\
\varepsilon_{\|} \\
\boldsymbol{\epsilon}_{b}
\end{array}\right\} \mathbf{C}_{\perp}\left\{\begin{array}{l}
\boldsymbol{\epsilon}_{m} \\
\varepsilon_{\|} \\
\boldsymbol{\epsilon}_{b}
\end{array}\right\}+\left\{\begin{array}{l}
\boldsymbol{\gamma}_{o} \\
\boldsymbol{\gamma}_{n}
\end{array}\right\}^{T} \mathbf{C}_{T}\left\{\begin{array}{l}
\boldsymbol{\gamma}_{o} \\
\boldsymbol{\gamma}_{n}
\end{array}\right\}\right\rangle-P^{e}
\end{aligned}
$$

in which

$$
\begin{aligned}
J_{o} & =\left.J\right|_{\zeta=0},\langle\circ\rangle=2 \int_{-1}^{+1} \int_{-1}^{+1} \circ J_{o} d \xi d \eta, \\
\mathbf{C}_{\perp} & =\frac{1}{2} \int_{-1}^{+1}\left[\begin{array}{ccc}
\mathbf{C}_{=} & \mathbf{C}_{\times} & \zeta \mathbf{C}_{=} \\
\mathbf{C}_{\times}^{T} & C_{\|} & \zeta \mathbf{C}_{\times}^{T} \\
\zeta \mathbf{C}_{=}^{T} & \zeta \mathbf{C}_{\times} & \zeta^{2} \mathbf{C}_{=}
\end{array}\right] d \zeta, \mathbf{C}_{T}=\frac{1}{2} \int_{-1}^{+1}\left[\begin{array}{cc}
\mathbf{C}_{t} & \zeta \mathbf{C}_{t} \\
\zeta \mathbf{C}_{t}^{T} & \zeta^{2} \mathbf{C}_{t}
\end{array}\right] d \zeta .
\end{aligned}
$$

By invoking (5), the element stiffness matrix is :

$$
\mathbb{K}=\left\langle\mathbb{B}_{\perp}^{T} \mathbf{C}_{\perp} \mathbb{B}_{\perp}+\mathbb{B}_{T}^{T} \mathbf{C}_{T} \mathbb{B}_{T}\right\rangle \quad \text { where } \quad \mathbb{B}_{\perp}=\left[\begin{array}{c}
\mathbb{B}_{m} \\
\mathbb{B}_{\|} \\
\mathbb{B}_{b}
\end{array}\right] \text { and } \quad \mathbb{B}_{T}=\left[\begin{array}{c}
\mathbb{B}_{o} \\
\mathbb{B}_{n}
\end{array}\right]
$$

Recalling that $\boldsymbol{\epsilon}_{m}, \varepsilon_{\|}, \boldsymbol{\epsilon}_{b}, \boldsymbol{\gamma}_{o}$ and $\boldsymbol{\gamma}_{n}$ are independent of $\zeta, \mathbf{C}_{\perp}$ and $\mathbf{C}_{T}$ relate the following 
generalized stresses to the generalized strains as :

$$
\begin{aligned}
& \left\{\begin{array}{l}
\mathbf{N} \\
T \\
\mathbf{M}
\end{array}\right\}=\frac{1}{2} \int_{-1}^{+1}\left\{\begin{array}{c}
\boldsymbol{\sigma}_{=} \\
\sigma_{\|} \\
\zeta \boldsymbol{\sigma}_{=}
\end{array}\right\} \mathrm{d} \zeta=\frac{1}{2} \int_{-1}^{+1}\left\{\begin{array}{c}
\mathbf{C}_{=}\left(\boldsymbol{\epsilon}_{m}+\zeta \boldsymbol{\epsilon}_{b}\right)+\mathbf{C}_{x} \varepsilon_{\|} \\
\mathbf{C}_{x}^{T}\left(\boldsymbol{\epsilon}_{m}+\zeta \boldsymbol{\epsilon}_{b}\right)+C_{\|} \varepsilon_{\|} \\
\zeta \mathbf{C}_{=}\left(\boldsymbol{\epsilon}_{m}+\zeta \boldsymbol{\epsilon}_{b}\right)+\zeta \mathbf{C}_{x} \varepsilon_{\|}
\end{array}\right\} \mathrm{d} \zeta=\mathbf{C}_{\perp}\left\{\begin{array}{c}
\boldsymbol{\epsilon}_{m} \\
\varepsilon_{\|} \\
\boldsymbol{\epsilon}_{b}
\end{array}\right\}, \\
& \left\{\begin{array}{l}
\mathbf{Q}_{o} \\
\mathbf{Q}_{n}
\end{array}\right\}=\frac{1}{2} \int_{-1}^{+1}\left\{\begin{array}{c}
\boldsymbol{\tau} \\
\zeta \boldsymbol{\tau}
\end{array}\right\} \mathrm{d} \zeta=\frac{1}{2} \int_{-1}^{+1}\left\{\begin{array}{c}
\mathbf{C}_{t}\left(\boldsymbol{\gamma}_{o}+\zeta \boldsymbol{\gamma}_{n}\right) \\
\zeta \mathbf{C}_{t}\left(\boldsymbol{\gamma}_{o}+\zeta \boldsymbol{\gamma}_{n}\right)
\end{array}\right\} \mathbf{C}_{t} \mathrm{~d} \zeta=\mathbf{C}_{T}\left\{\begin{array}{l}
\boldsymbol{\gamma}_{o} \\
\boldsymbol{\gamma}_{n}
\end{array}\right\} .
\end{aligned}
$$

In particular, $\mathbf{N}, T$ and $\mathbf{M}$ are respectively the membrane stress, the thickness-average of the thickness stress and the bending stresses. $\mathbf{C}_{\perp}$ will be termed as the generalized laminate stiffness matrix.

\section{PLANE STRESS ENFORCEMENT \& ENHANCED ASSUMED STRAIN METHOD}

In this session, thickness locking and the drawbacks of its existing remedies in laminate analyses are discussed. For homogeneous materials, the generalized laminate stiffness matrix in (8) can be evaluated to be :

$$
\mathbf{C}_{\perp}=\left[\begin{array}{ccc}
\mathbf{C}_{=} & \mathbf{C}_{\times} & \mathbf{0} \\
\mathbf{C}_{\times}^{T} & C_{\|} & \mathbf{0} \\
\mathbf{0} & \mathbf{0} & \mathbf{C}_{=} / 3
\end{array}\right] .
$$

When $\mathbf{N}=\mathbf{0}$ and $\mathrm{T}=0$ are prescribed, we have

$$
\boldsymbol{\epsilon}_{m}=\mathbf{0}, \varepsilon_{\|}=0, \boldsymbol{\epsilon}_{b}=3 \mathbf{C}_{=}^{-1} \mathbf{M} \text { and } \sigma_{\|}=\zeta \mathbf{C}_{\times}^{T} \boldsymbol{\epsilon}_{b} .
$$

Unless the Poisson's ratio vanishes, magnitude of the above bending strain is always smaller than the exact bending strain $\boldsymbol{\epsilon}_{b}^{\text {exact }}=3 \mathbf{S}_{=} \mathbf{M}$ where $\mathbf{S}_{=}$is the plane stress compliance matrix, see (6). Hence, the bending deflection predicted by $\mathbb{K}$ or using $\mathbf{C}_{\perp}$ is smaller than the exact solution. Moreover, a plane strain state $\left(\varepsilon_{\|}=0\right)$ instead of the plane stress state $\left(\sigma_{\|}=0\right)$ is predicted. These phenomena are sometimes referred to as thickness locking.

The plane stress enforcement $[1,2,4,7,8]$ resolve the thickness locking by changing the constitutive relation to :

$$
\left\{\begin{array}{c}
\boldsymbol{\sigma}_{=} \\
\hdashline \sigma_{\|}
\end{array}\right\}=\left[\begin{array}{c:c}
\mathbf{S}_{=}^{-1} & \mathbf{0}_{1 \times 3} \\
\hdashline \mathbf{0}_{3 \times 1} & 1 / S_{\|}
\end{array}\right]\left\{\begin{array}{l}
\boldsymbol{\epsilon}_{=} \\
\hdashline \varepsilon_{\|}
\end{array}\right\}
$$


which leads to the following plane stress generalized laminate stiffness matrix and element stiffness matrix :

$$
\mathbf{C}_{\perp}^{P S}=\frac{1}{2} \int_{-1}^{+1}\left[\begin{array}{ccc}
\mathbf{S}_{=}^{-1} & \mathbf{0} & \zeta \mathbf{S}_{=}^{-1} \\
\mathbf{0} & 1 / S_{\|} & \mathbf{0} \\
\zeta \mathbf{S}_{=}^{-1} & \mathbf{0} & \zeta^{2} \mathbf{S}_{=}^{-1}
\end{array}\right] d \zeta \quad \text { and } \quad \mathbb{\mathbb { S } ^ { P S }}=\left\langle\mathbb{B}_{\perp}^{T} \mathbf{C}_{\perp}^{P S} \mathbb{B}_{\perp}+\mathbb{B}_{T}^{T} \mathbf{C}_{T} \mathbb{B}_{T}\right\rangle
$$

As the inplane and out-of-plane normal stress-strain response are decoupled, the assumption gives $\sigma_{\|}=\varepsilon_{\|}=0$. On the other hand, the enhanced assumed strain method [5,6] enriches the thickness strain with linear $\zeta$-terms. In other words, we have

$$
\sigma_{\|}=\mathbf{C}_{\times}^{T}\left(\zeta \epsilon_{b}\right)+\mathbf{C}_{\|}\left(\varepsilon_{\|}+\zeta \varepsilon_{\|}^{E A S}\right)
$$

such that $\sigma_{\|}=0$ becomes possible for $\boldsymbol{\epsilon}_{b} \neq \mathbf{0}$. Meanwhile, the hybrid-stress overcomes thickness locking by assuming $\sigma_{\|}$to be independent of $\zeta[3,12]$. The displacement-derived thickness stress in (12) will vanish in the assumed stress field.

The two problems depicted in Figure 2 are considered by the conventional, plane-stress (along z-direction) enforcement, enhanced assumed strain and hybrid-stress six-node plane elements which are the two-dimensional counterparts of the eighteen-node solid-shell elements. The rectangular block is modelled by one element and comprised of two plies of isotropic materials designated as $B$ (bottom) and $T$ (top) for $z<0$ and $z>0$, respectively. The block is subjected to uniform compressive stress and end moment. Plane stress condition is assumed along the y-direction. After examining the displacement-derived strains, the following enhanced assumed strain and hybridstress modes are employed :

$$
\boldsymbol{\epsilon}^{E A S}=\left\{\begin{array}{c}
\varepsilon_{x}^{E A S} \\
\varepsilon_{z}^{E A S} \\
2 \varepsilon_{z x}^{E A S}
\end{array}\right\}=\left[\begin{array}{ll}
0 & 0 \\
1 & \xi \\
0 & 0
\end{array}\right]\left\{\begin{array}{l}
\alpha_{1} \\
\alpha_{2}
\end{array}\right\} \text { and } \boldsymbol{\sigma}=\left\{\begin{array}{c}
\sigma_{x} \\
\sigma_{z} \\
\tau_{x y}
\end{array}\right\}=\left[\begin{array}{ccccccccc}
1 & \xi & \zeta & \zeta \xi & 0 & 0 & 0 & 0 & 0 \\
0 & 0 & 0 & 0 & 1 & \xi & \xi^{2} & 0 & 0 \\
0 & 0 & 0 & 0 & 0 & 0 & 0 & 1 & \xi
\end{array}\right]\left\{\begin{array}{c}
\beta_{1} \\
\vdots \\
\beta_{9}
\end{array}\right\}
$$

in the respective elements. For homogeneous materials, both elements yield the exact stresses and displacements in the two problems in Figure 2. On the other hand, Tables 1a and $1 \mathrm{~b}$ list the deflections and thickness stresses when the elements are laminated. None of the conventional, plane stress, enhanced assumed strain and hybrid-stress elements can deliver accurate deflections and thickness stresses simultaneously in all cases.

Table 1a: Deflections and thickness stresses of the rectangular block under compression (Figure 2a)

\begin{tabular}{|c|c|c|c|c|c|c|}
\hline \multirow{2}{*}{ formulation } & \multicolumn{3}{|c|}{$E_{B}=1, E_{T}=10, v_{B}=0, v_{T}=0$} & \multicolumn{3}{c|}{$E_{B}=1, E_{T}=10, v_{B}=0.2, v_{T}=0.3$} \\
\cline { 2 - 7 } & deflection & $\sigma_{z}$ for $\mathrm{z}<0$ & $\sigma_{z}$ for $\mathrm{z}>0$ & deflection & $\sigma_{z}$ for $\mathrm{z}<0$ & $\sigma_{z}$ for $\mathrm{z}>0$ \\
\hline
\end{tabular}




\begin{tabular}{|c|c|c|c|c|c|c|}
\hline conventional & -0.3636 & -0.1818 & -1.8181 & -0.3325 & -0.1731 & -1.8268 \\
\hline plane stress & -0.3636 & -0.1818 & -1.8181 & -0.3636 & -0.1818 & -1.8182 \\
\hline EAS & -0.7303 & $\begin{array}{c}-0.3651 \\
+0.4481 \mathrm{z}\end{array}$ & $\begin{array}{c}-3.6515 \\
+4.4813 \mathrm{z}\end{array}$ & -0.6824 & $\begin{array}{c}-0.3554 \\
+0.4408 z\end{array}$ & $\begin{array}{c}-3.7492 \\
+4.6499 z\end{array}$ \\
\hline hybrid stress & exact & exact & exact & 1.0515 & exact & exact \\
\hline$\tilde{\mathbf{C}}_{\perp}$ or $\tilde{\mathbb{K}}$ & exact & exact & exact & exact & exact & exact \\
\hline analytical & -1.1 & -1 & -1 & 1.051 & -1 & -1 \\
\hline
\end{tabular}

Table $1 \mathrm{~b}$ : Deflections at point A and thickness stresses of the rectangular block under bending (Figure 2b)

\begin{tabular}{|c|c|c|c|c|c|c|}
\hline \multirow{2}{*}{ formulation } & \multicolumn{3}{|c|}{$E_{B}=1, E_{T}=10, v_{B}=0, v_{T}=0$} & \multicolumn{3}{c|}{$E_{B}=1, E_{T}=10, v_{B}=0.2, v_{T}=0.3$} \\
\cline { 2 - 7 } & deflection & $\sigma_{z}$ for $\mathrm{z}<0$ & $\sigma_{z}$ for $\mathrm{z}>0$ & deflection & $\sigma_{z}$ for $\mathrm{z}<0$ & $\sigma_{z}$ for $\mathrm{z}>0$ \\
\hline conventional & exact & exact & exact & 25.5989 & $\begin{array}{c}0.0484 \\
-0.1066 z\end{array}$ & $\begin{array}{c}0.7422 \\
-1.6878 z\end{array}$ \\
\hline plane stress & exact & exact & exact & exact & exact & exact \\
\hline EAS & exact & exact & exact & 27.3246 & $\begin{array}{c}-0.0073 \\
+0.0292 z\end{array}$ & $\begin{array}{c}0.1681 \\
-0.2924 z\end{array}$ \\
\hline hybrid stress & 41.25 & exact & exact & 41.25 & exact & exact \\
\hline$\tilde{\mathbf{C}}_{\perp}$ or $\tilde{\mathbb{K}}$ & exact & exact & exact & exact & exact & exact \\
\hline analytical & 27.3859 & 0 & 0 & 27.3859 & 0 & 0 \\
\hline
\end{tabular}

* based on Classical Lamination Plate Theory

\section{MODIFIED GENERALIZED LAMINATE STIFFNESS MATRIX}

Despite of the erroneous bending deflections predicted by the hybrid-stress element, the predicted thickness stresses are exact in all cases. Moreover, the traction reciprocity condition at the ply interface is satisfied with the thickness stress taken to be indepedent of $\zeta$. A new method to overcome thickness locking and the abnormalities in laminate analyses is here proposed. The constitutive relation in (6) is first rewritten as :

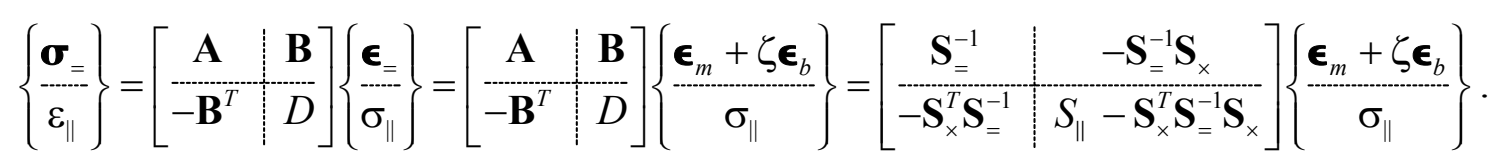

By assuming the element thickness stress to be independent of $\zeta$, the above equation leads to

$$
\left\{\begin{array}{l}
\mathbf{N} \\
\bar{\varepsilon}_{\|} \\
\mathbf{M}
\end{array}\right\}=\frac{1}{2} \int_{-1}^{+1}\left\{\begin{array}{c}
\boldsymbol{\sigma}_{=} \\
\varepsilon_{\|} \\
\zeta \boldsymbol{\sigma}_{=}
\end{array}\right\} \mathrm{d} \zeta=\frac{1}{2} \int_{-1}^{+1}\left[\begin{array}{cc}
\mathbf{A} & \mathbf{B} \\
-\mathbf{B}^{T} & D \\
\zeta \mathbf{A} & \zeta \mathbf{B}
\end{array}\right]\left\{\begin{array}{c}
\boldsymbol{\epsilon}_{m}+\zeta \boldsymbol{\epsilon}_{b} \\
\hdashline \sigma_{\|}
\end{array}\right\} \mathrm{d} \zeta=\left[\begin{array}{ccc}
\mathbf{A}_{0} & \mathbf{B}_{0} & \mathbf{A}_{1} \\
-\mathbf{B}_{0}^{T} & D_{0} & -\mathbf{B}_{1}^{T} \\
\mathbf{A}_{1} & \mathbf{B}_{1} & \mathbf{A}_{2}
\end{array}\right]\left\{\begin{array}{c}
\boldsymbol{\epsilon}_{m} \\
\sigma_{\|} \\
\boldsymbol{\epsilon}_{b}
\end{array}\right\}
$$

where

$\bar{\varepsilon}_{\| \mid}$is the average thickness strain, $\left[\mathbf{A}_{0}, \mathbf{A}_{1}, \mathbf{A}_{2}, \mathbf{B}_{0}, \mathbf{B}_{1}, D_{0}\right]=\frac{1}{2} \int_{-1}^{+1}\left[\mathbf{A}, \zeta \mathbf{A}, \zeta^{2} \mathbf{A}, \mathbf{B}, \zeta \mathbf{B}, D\right] \mathrm{d} \zeta$. 
By changing the equation objects of (17), we have

$$
\left\{\begin{array}{l}
\mathbf{N} \\
\sigma_{\|} \\
\mathbf{M}
\end{array}\right\}=\left[\begin{array}{ccc}
\mathbf{A}_{0}+\mathbf{B}_{0} \mathbf{B}_{0}^{T} / D_{0} & \mathbf{B}_{0} / D_{0} & \mathbf{A}_{1}+\mathbf{B}_{0} \mathbf{B}_{1}^{T} / D_{0} \\
\mathbf{B}_{0}^{T} / D_{0} & 1 / D_{0} & \mathbf{B}_{1}^{T} / D_{0} \\
\mathbf{A}_{1}+\mathbf{B}_{1} \mathbf{B}_{0}^{T} / D_{0} & \mathbf{B}_{1} / D_{0} & \mathbf{A}_{2}+\mathbf{B}_{1} \mathbf{B}_{1}^{T} / D_{0}
\end{array}\right]\left\{\begin{array}{l}
\boldsymbol{\epsilon}_{m} \\
\bar{\varepsilon}_{\|} \\
\boldsymbol{\epsilon}_{b}
\end{array}\right\}=\tilde{\mathbf{C}}_{\perp}\left\{\begin{array}{l}
\boldsymbol{\epsilon}_{m} \\
\bar{\varepsilon}_{\|} \\
\boldsymbol{\epsilon}_{b}
\end{array}\right\} .
$$

The matrix $\tilde{\mathbf{C}}_{\perp}$ will be termed as the modified laminate stiffness matrix.

We then recall that the thickness strain for elements possessing only two layers of nodes can only be the thickness-average of its pointwise value. In this context, the second strain-displacement relation in (5) can be re-written as :

$$
\bar{\varepsilon}_{\|}=\mathbb{B}_{\|} \mathbf{q}
$$

By introducing $\bar{\varepsilon}_{\|}$and $\tilde{\mathbf{C}}_{\perp},(8)$ is modified to be

$$
\Pi_{m P}^{e}=\frac{1}{2}\left\langle\left\{\begin{array}{l}
\boldsymbol{\epsilon}_{m} \\
\bar{\varepsilon}_{\|} \\
\boldsymbol{\epsilon}_{b}
\end{array}\right\}^{T} \tilde{\mathbf{C}}_{\perp}\left\{\begin{array}{l}
\boldsymbol{\epsilon}_{m} \\
\bar{\varepsilon}_{\|} \\
\boldsymbol{\epsilon}_{b}
\end{array}\right\}+\left\{\begin{array}{l}
\boldsymbol{\gamma}_{o} \\
\boldsymbol{\gamma}_{n}
\end{array}\right\}^{T} \mathbf{C}_{T}\left\{\begin{array}{l}
\boldsymbol{\gamma}_{o} \\
\boldsymbol{\gamma}_{n}
\end{array}\right\}\right\rangle-P^{e}
$$

which leads to the following element :

$$
\tilde{\mathbb{K}}=\left\langle\mathbb{B}_{\perp}^{T} \tilde{\mathbf{C}}_{\perp} \mathbb{B}_{\perp}+\mathbb{B}_{T}^{T} \mathbf{C}_{T} \mathbb{B}_{T}\right\rangle .
$$

In the post-processing stage, the thickness stress should first be computed from (18) before the inplane stress is computed by (16). The transverse shear stress can be calculated in the conventional manner.

As noted in Tables 1a and 1b, the abnormalities bothering the conventional, plane stress, enhanced assumed strain and hybrid stress elements are not observed in the element that employs $\tilde{\mathbf{C}}_{\perp}$. Despite of the above successes, the fully integrated eighteen-node $\tilde{\mathbb{K}}$ suffers from shear and membrane lockings whereas the reduced integrated $\tilde{\mathbb{K}}$ is plagued by commutable mechanisms. A stabilization procedure will be formulated to suppress the commutable mechanisms of the reduced integrated element via a modified hybrid variational functional. It should be remarked that trapezoidal locking occurs in linear elements such as eight-node hexahedrons but not in the present quadratic element.

\section{ELEMENT FORMULATION USING A GENERALIZED HYBRID FUNCTIONAL}

By introducing the generalized stresses $\mathbf{N}, \sigma_{\|}, \mathbf{M}$, and $\mathbf{Q}$ 's as independently assumed field variables 
in (20), the following modified elementwise Hellinger-Reissner functional is obtained :

$$
\Pi^{e}=\left\langle-\frac{1}{2}\left\{\begin{array}{c}
\mathbf{N} \\
\sigma_{\|} \\
\mathbf{M}
\end{array}\right\}^{T} \tilde{\mathbf{C}}_{\perp}^{-1}\left\{\begin{array}{c}
\mathbf{N} \\
\sigma_{\|} \\
\mathbf{M}
\end{array}\right\}+\left\{\begin{array}{c}
\mathbf{N} \\
\sigma_{\|} \\
\mathbf{M}
\end{array}\right\}^{T}\left\{\begin{array}{l}
\boldsymbol{\epsilon}_{m} \\
\bar{\varepsilon}_{\|} \\
\mathbf{\epsilon}_{b}
\end{array}\right\}-\frac{1}{2}\left\{\begin{array}{l}
\mathbf{Q}_{o} \\
\mathbf{Q}_{n}
\end{array}\right\}^{T} \mathbf{C}_{T}^{-1}\left\{\begin{array}{l}
\mathbf{Q}_{o} \\
\mathbf{Q}_{n}
\end{array}\right\}+\left\{\begin{array}{l}
\mathbf{Q}_{o} \\
\mathbf{Q}_{n}
\end{array}\right\}^{T}\left\{\begin{array}{l}
\boldsymbol{\gamma}_{o} \\
\boldsymbol{\gamma}_{n}
\end{array}\right\}\right\rangle-P^{e}
$$

We assume that the generalized stresses can be partitioned into lower " $L$ " and higher " $H$ " order modes and expressed as :

$$
\left\{\begin{array}{l}
\mathbf{N} \\
\sigma_{\|} \\
\mathbf{M}
\end{array}\right\}=\left\{\begin{array}{l}
\mathbf{N} \\
\sigma_{\|} \\
\mathbf{M}
\end{array}\right\}_{L}+\left\{\begin{array}{l}
\mathbf{N} \\
\sigma_{\|} \\
\mathbf{M}
\end{array}\right\}_{H}=\mathbb{P}_{\perp L} \boldsymbol{\beta}_{\perp L}+\mathbb{P}_{\perp H} \boldsymbol{\beta}_{\perp H},\left\{\begin{array}{l}
\mathbf{Q}_{o} \\
\mathbf{Q}_{n}
\end{array}\right\}=\left\{\begin{array}{l}
\mathbf{Q}_{o} \\
\mathbf{Q}_{n}
\end{array}\right\}_{L}+\left\{\begin{array}{l}
\mathbf{Q}_{o} \\
\mathbf{Q}_{n}
\end{array}\right\}_{H}=\mathbb{P}_{T L} \boldsymbol{\beta}_{T L}+\mathbb{P}_{T H} \boldsymbol{\beta}_{T H}
$$

where

P's are the shape function matrices, $\boldsymbol{\beta}$ 's are the vectors of coefficients,

$$
\mathbb{P}_{\perp L}=\left[\begin{array}{llll}
\mathbf{I}_{7} & \xi \mathbf{I}_{7} & \eta \mathbf{I}_{7} & \xi \eta \mathbf{I}_{7}
\end{array}\right], \mathbb{P}_{T L}=\left[\begin{array}{llll}
\mathbf{I}_{4} & \xi \mathbf{I}_{4} & \eta \mathbf{I}_{4} & \xi \eta \mathbf{I}_{4}
\end{array}\right]
$$

Moreover,

$$
\begin{aligned}
& \left.\mathbb{P}_{\perp H}\right|_{\xi= \pm 1 / \sqrt{3}, \mathfrak{\eta}= \pm 1 / \sqrt{3}}=\mathbf{0},\left.\mathbb{P}_{T H}\right|_{\xi= \pm 1 / \sqrt{3}, \eta= \pm 1 / \sqrt{3}}=\mathbf{0} \\
& \left\langle\mathbb{P}_{\perp L}^{T} \tilde{\mathbf{C}}_{\perp}^{-1} \mathbb{P}_{\perp H}\right\rangle=\mathbf{0} \text { and }\left\langle\mathbb{P}_{T L}^{T} \mathbf{C}_{T}^{-1} \mathbb{P}_{T H}\right\rangle=\mathbf{0}
\end{aligned}
$$

The last two expressions are the orthogonality conditions on the lower and higher order generalized stress modes [7,15]. After substituting (5), (19), (23) and (24) into (22), we have

$$
\Pi^{e}=-\frac{1}{2}\left(\boldsymbol{\beta}_{\perp L}^{T} \mathbb{M}_{\perp L} \boldsymbol{\beta}_{\perp L}+\boldsymbol{\beta}_{\perp H}^{T} \mathbb{U}_{\perp H} \boldsymbol{\beta}_{\perp H}+\boldsymbol{\beta}_{T L}^{T} \mathbb{H}_{T L} \boldsymbol{\beta}_{T L}+\boldsymbol{\beta}_{T H}^{T} \mathbb{M}_{T H} \boldsymbol{\beta}_{T H}\right)
$$

$$
+\left(\boldsymbol{\beta}_{\perp L}^{T} \mathbb{G}_{\perp L}+\boldsymbol{\beta}_{\perp H}^{T} \mathbb{G}_{\perp H}+\boldsymbol{\beta}_{T L}^{T} \mathbb{G}_{T L}+\boldsymbol{\beta}_{T H}^{T} \mathbb{G}_{T H}\right) \mathbf{q}-P^{e}
$$

In the functional, the flexibility matrices $\mathbb{H}$ 's and leverage matrices $\mathbb{G}^{\prime}$ 's are defined as :

$$
\begin{aligned}
& \mathbb{H}_{\perp L}=\left\langle\mathbb{P}_{\perp L}^{T} \tilde{\mathbf{C}}_{\perp}^{-1} \mathbb{P}_{\perp L}\right\rangle_{L}, \mathbb{H}_{\perp H}=\left\langle\mathbb{P}_{\perp H}^{T} \tilde{\mathbf{C}}_{\perp}^{-1} \mathbb{P}_{\perp H}\right\rangle_{H}, \mathbb{H}_{T L}=\left\langle\mathbb{P}_{T L}^{T} \mathbf{C}_{T}^{-1} \mathbb{P}_{T L}\right\rangle_{L}, \mathbb{H}_{T H}=\left\langle\mathbb{P}_{T H}^{T} \mathbf{C}_{T}^{-1} \mathbb{P}_{T H}\right\rangle_{H}, \\
& \mathbb{G}_{\perp L}=\left\langle\mathbb{P}_{\perp L}^{T} \mathbb{B}_{\perp}\right\rangle_{L}, \mathbb{G}_{\perp H}=\left\langle\mathbb{P}_{\perp H}^{T} \mathbb{B}_{\perp}\right\rangle_{H}, \mathbb{G}_{T L}=\left\langle\mathbb{P}_{T L}^{T} \mathbb{B}_{T}\right\rangle_{L}, \mathbb{G}_{T H}=\left\langle\mathbb{P}_{T H}^{T} \mathbb{B}_{T}\right\rangle_{H}
\end{aligned}
$$

The subscripts " $L$ " and " $H$ " to the integral operator \langle\rangle indicate that the standard order of integration [16] is the second (lower) and third (higher) order quadratures, respectively. Furthermore, all integrations are conducted by using the standard orders of integration. The stationary nature of the functional with respect to $\boldsymbol{\beta}$ 's gives :

$$
\mathbb{H}_{\perp L} \boldsymbol{\beta}_{\perp L}=\mathbb{G}_{\perp L} \mathbf{q}, \mathbb{H}_{\perp H} \boldsymbol{\beta}_{\perp H}=\mathbb{G}_{\perp H} \mathbf{q}, \mathbb{H}_{T L} \boldsymbol{\beta}_{T L}=\mathbb{G}_{T L} \mathbf{q}, \mathbb{U}_{T H} \boldsymbol{\beta}_{T H}=\mathbb{G}_{T H} \mathbf{q}
$$

with which (25) becomes : 


$$
\Pi^{e}=\frac{1}{2} \mathbf{q}^{T}\left(\mathbb{G}_{\perp L}^{T} \mathbb{H}_{\perp L}^{-1} \mathbb{G}_{\perp L}+\mathbb{G}_{\perp H}^{T} \mathbb{M}_{\perp H}^{-1} \mathbb{G}_{\perp H}+\mathbb{G}_{T L}^{T} \mathbb{H}_{T L}^{-1} \mathbb{G}_{T L}+\mathbb{G}_{T H}^{T} \mathbb{M}_{T H}^{-1} \mathbb{G}_{T H}\right) \mathbf{q}-P^{e}
$$

It can be proved that at the second order quadrature point where $\mathbb{P}_{\perp H}$ and $\mathbb{P}_{T H}$ vanish, the generalized stresses derived from $\boldsymbol{\beta}$ 's and the element displacement $\mathbf{q}$ are identical [7,15], i.e.

$$
\left\{\begin{array}{l}
\mathbf{N} \\
\sigma_{\|} \\
\mathbf{M}
\end{array}\right\}=\mathbb{P}_{\perp L} \boldsymbol{\beta}_{\perp L}=\mathbb{P}_{\perp L} \mathbb{H}_{\perp L}^{-1} \mathbb{G}_{\perp L} \mathbf{q}=\tilde{\mathbf{C}}_{\perp} \mathbb{B}_{\perp} \mathbf{q},\left\{\begin{array}{l}
\mathbf{Q}_{o} \\
\mathbf{Q}_{n}
\end{array}\right\}=\mathbb{P}_{T L} \boldsymbol{\beta}_{T L}=\mathbb{P}_{T L} \mathbb{H}_{T L}^{-1} \mathbb{G}_{T L} \mathbf{q}=\mathbf{C}_{T} \mathbb{B}_{T} \mathbf{q}
$$

It can also be shown by simply linear algebra that

$$
\mathbb{G}_{\perp L}^{T} \mathbb{M}_{\perp L}^{-1} \mathbb{G}_{\perp L}+\mathbb{G}_{T L}^{T} \mathbb{\boxplus}_{T L}^{-1} \mathbb{G}_{T L}=\left\langle\mathbb{B}_{\perp}^{T} \tilde{\mathbf{C}}_{\perp} \mathbb{B}_{\perp}+\mathbb{B}_{T}^{T} \mathbf{C}_{T} \mathbb{B}_{T}\right\rangle_{L}=\tilde{\mathbb{K}}_{L}
$$

where $\tilde{\mathbb{K}}_{L}$ is the reduced integrated version of $\tilde{\mathbb{K}}$ in (21). Thus, (28) can be re-written as :

$$
\Pi^{e}=\frac{1}{2} \mathbf{q}^{T}\left(\tilde{\mathbb{K}}_{L}+\mathbb{G}_{\perp H}^{T} \mathbb{W}_{\perp H}^{-1} \mathbb{G}_{\perp H}+\mathbb{G}_{T H}^{T} \mathbb{M}_{T H}^{-1} \mathbb{G}_{T H}\right) \mathbf{q}-P^{e}
$$

In other words, $\mathbb{G}_{\perp H}^{T} \mathbb{H}_{\perp H}^{-1} \mathbb{G}_{\perp H}$ and $\mathbb{G}_{T H}^{T} \mathbb{W}_{T H}^{-1} \mathbb{G}_{T H}$ play the role of stabilizing $\tilde{\mathbb{K}}_{L}$.

\section{HIGHER ORDER MODES FOR ASSUMED STRESS RESULTANTS}

For a geometrically regular reduced integrated element with its natural co-ordinates parallel to the global Cartesian co-ordinates, the commutable and incommutable mechanisms or zero energy modes are $[1,2,7]$ :

$$
\left\{\begin{array}{l}
U_{\xi} \\
U_{\eta} \\
U_{\zeta}
\end{array}\right\}_{\text {commutable }}=p_{\xi \eta}\left[\begin{array}{cccccc}
1 & \zeta & 0 & 0 & 0 & 0 \\
0 & 0 & 1 & \zeta & 0 & 0 \\
0 & 0 & 0 & 0 & 1 & \zeta
\end{array}\right]\left\{\begin{array}{c}
c_{1} \\
\vdots \\
c_{6}
\end{array}\right\},\left\{\begin{array}{l}
U_{\xi} \\
U_{\eta} \\
U_{\zeta}
\end{array}\right\} \quad=\left[\begin{array}{cc}
p_{\xi} & \zeta p_{\xi} \\
-p_{\eta} & -\zeta p_{\eta} \\
0 & 0
\end{array}\right]\left\{\begin{array}{l}
c_{7} \\
c_{8}
\end{array}\right\}
$$

in which $p_{\xi}=\xi\left(3 \eta^{2}-1\right), p_{\eta}=\eta\left(3 \xi^{2}-1\right), p_{\xi \eta}=\left(3 \xi^{2}-1\right)\left(3 \eta^{2}-1\right)$ and $c_{i}$ 's are coefficients. The incommutable mechanisms are suppressed by the presence of adjacent elements and, thus, can be ignored in real analyses. However, the commutable mechanisms may propagate in a mesh and should be stabilized. The derived covariant strain from the commutable mechanisms is : 


$$
\left\{\begin{array}{c}
\varepsilon_{\xi} \\
\varepsilon_{\eta} \\
\varepsilon_{\zeta} \\
\gamma_{\zeta \eta} \\
\gamma_{\zeta \xi} \\
\gamma_{\xi \eta}
\end{array}\right\}=\left[\begin{array}{cccccc}
6 p_{\xi} & 6 \zeta p_{\xi} & 0 & 0 & 0 & 0 \\
0 & 0 & 6 p_{\eta} & 6 \zeta p_{\eta} & 0 & 0 \\
0 & 0 & 0 & 0 & 0 & p_{\xi \eta} \\
0 & 0 & 0 & p_{\xi \eta} & 6 p_{\eta} & 6 \zeta p_{\eta} \\
0 & p_{\xi \eta} & 0 & 0 & 6 p_{\xi} & 6 \zeta p_{\xi} \\
6 p_{\eta} & 6 \zeta p_{\eta} & 6 p_{\xi} & 6 \zeta p_{\xi} & 0 & 0
\end{array}\right]\left\{\begin{array}{l}
c_{1} \\
c_{2} \\
c_{3} \\
c_{4} \\
c_{5} \\
c_{6}
\end{array}\right\}
$$

As $p$ 's vanish at all the second order quadrature points, the above strain modes do not induce any strain energy in the reduced integrated element. To stabilize them, the higher order generalized stress modes must be chosen such that their inner products with the strain modes derived from the mechanisms must not be zero. In this light, the following higher order generalized stress modes can be selected by modifying the strain modes :

$$
\left\{\begin{array}{l}
\mathbf{N} \\
\sigma_{\|} \\
\mathbf{M}
\end{array}\right\}_{H}=\mathbb{P}_{\perp H} \boldsymbol{\beta}_{\perp H}=\frac{1}{J_{o}}\left[\begin{array}{c|c}
\mathbf{T}_{\varepsilon} \mathbf{P}_{\perp} & \mathbf{0}_{3 \times 2} \\
\hdashline \mathbf{0}_{1 \times 2} & \mathbf{0}_{1 \times 2} \\
\hdashline \mathbf{0}_{3 \times 2} & \mathbf{T}_{\varepsilon} \mathbf{P}_{\perp}
\end{array}\right] \boldsymbol{\beta}_{\perp H},\left\{\begin{array}{l}
\mathbf{Q}_{o} \\
\mathbf{Q}_{n}
\end{array}\right\}_{H}=\mathbb{P}_{T H} \boldsymbol{\beta}_{T H}=\frac{1}{J_{o}}\left[\begin{array}{c:c}
\mathbf{T}_{\gamma} \mathbf{P}_{T} & \mathbf{0}_{2 \times 1} \\
\mathbf{0}_{2 \times 1} & \mathbf{T}_{\gamma} \mathbf{P}_{T}
\end{array}\right] \boldsymbol{\beta}_{T H}
$$

where

$$
\mathbf{P}_{\perp}=\left[\begin{array}{cc}
p_{\xi} & 0 \\
0 & p_{\eta} \\
0 & 0
\end{array}\right], \mathbf{P}_{T}=\left[\begin{array}{c}
p_{\xi} \\
p_{\eta}
\end{array}\right]
$$

Matrices $T_{\varepsilon}$ and $T_{\gamma}$ have been defined in (4). They transform the contravariant stress components into Cartesian stress components. The reciprocal of $J_{o}$ in $\mathbf{P}_{\perp}$ and $\mathbf{P}_{T}$ is important in securing the patch test fulfillment and simplifying the formulation of the higher order leverage matrices [15]. Following the definitions in (26) and consolidating (3), (5) and (34), we have

$$
\begin{aligned}
& \mathbb{G}_{\perp H} \mathbf{q}=\left\langle\mathbb{P}_{\perp H}^{T} \mathbb{B}_{\perp}\right\rangle \mathbf{q}=2 \int_{-1-1}^{+1+1}\left\{\begin{array}{l}
p_{\xi} \varepsilon_{\xi m}^{e} \\
p_{\eta} \varepsilon_{\eta m}^{e} \\
p_{\xi} \varepsilon_{\xi b}^{e} \\
p_{\eta} \varepsilon_{\eta b}^{e}
\end{array}\right\} d \xi d \eta=\left[\begin{array}{c}
\mathbb{G}_{m 1} \\
\mathbb{G}_{m 2} \\
\mathbb{G}_{b 1} \\
\mathbb{G}_{b 2}
\end{array}\right] \mathbf{q}, \\
& \mathbb{G}_{T H} \mathbf{q}=\left\langle\mathbb{P}_{T H}^{T} \mathbb{B}_{T}\right\rangle \mathbf{q}=2 \int_{-1}^{+1+1} \int_{-1}^{+1}\left\{\begin{array}{l}
p_{\xi} \gamma_{\xi \xi o}^{e}+p_{\eta} \gamma_{\xi \eta o}^{e} \\
p_{\xi} \gamma_{\xi \xi \eta}^{e}+p_{\eta} \gamma_{\xi \eta n}^{e}
\end{array}\right\} d \xi d \eta=\left[\begin{array}{c}
\mathbb{G}_{0} \\
\mathbb{G}_{n}
\end{array}\right] \mathbf{q} .
\end{aligned}
$$

Further expansion gives :

$$
\begin{aligned}
& \mathbb{G}_{m 1} \mathbf{q}=\mathbf{X}_{o}^{T} \mathbb{M}_{1} \mathbf{q}_{o}, \mathbb{G}_{m 2} \mathbf{q}=\mathbf{X}_{o}^{T} \mathbb{M}_{2} \mathbf{q}_{o}, \mathbb{G}_{b 1} \mathbf{q}=\mathbf{X}_{n}^{T} \mathbb{M}_{1} \mathbf{q}_{o}+\mathbf{X}_{o}^{T} \mathbb{M}_{1} \mathbf{q}_{n}, \\
& \mathbb{G}_{b 2} \mathbf{q}=\mathbf{X}_{n}^{T} \mathbb{M}_{2} \mathbf{q}_{o}+\mathbf{X}_{o}^{T} \mathbb{M}_{2} \mathbf{q}_{n}, \mathbb{G}_{o} \mathbf{q}=\mathbf{X}_{n}^{T} \mathbb{M}_{3} \mathbf{q}_{o}+\mathbf{X}_{o}^{T} \mathbb{M}_{4} \mathbf{q}_{n}, \mathbb{G}_{n} \mathbf{q}=\mathbf{X}_{n}^{T} \mathbb{M}_{3} \mathbf{q}_{n}+\mathbf{X}_{n}^{T} \mathbb{M}_{4} \mathbf{q}_{n}
\end{aligned}
$$


where

$$
\begin{aligned}
& \mathbb{M}_{1}=2 \int_{-1}^{+1} \int_{-1}^{+1} p_{\xi} \mathbb{N}_{, \xi}^{T} \mathbb{N}_{, \xi} d \xi d \eta, \mathbb{M}_{2}=2 \int_{-1}^{+1} \int_{-1}^{+1} p_{\eta} \mathbb{N}_{, \eta}^{T} \mathbb{N}_{, \eta} d \xi d \eta \\
& \mathbb{M}_{3}=2 \int_{-1}^{+1} \int_{-1}^{+1}\left(p_{\xi} \mathbb{N}^{T} \mathbb{N}_{, \xi}+p_{\eta} \mathbb{N}^{T} \mathbb{N}_{, \eta}\right) d \xi d \eta, \mathbb{M}_{4}=2 \int_{-1}^{+1+1} \int_{-1}^{+1}\left(p_{\xi} \mathbb{N}_{, \xi}^{T} \mathbb{N}+p_{\eta} \mathbb{N}_{, \eta}^{T} \mathbb{N}\right) d \xi d \eta
\end{aligned}
$$

It should be remarked that M's are independent of the nodal coordinates and can be programmed explicitly without resorting to any integration loop [7]. For the stress shape function matrix given in (34), the flexibility matrix can be manipulated as :

$$
\begin{aligned}
& \mathbb{W}_{\perp H}=\left\langle\mathbb{P}_{\perp H}^{T} \tilde{\mathbf{C}}_{\perp}^{-1} \mathbb{P}_{\perp H}\right\rangle=\left\langle\frac{1}{J_{o}^{2}}\left[\begin{array}{cc}
\mathbf{P}_{\perp} & \mathbf{0}_{3 \times 2} \\
\mathbf{0}_{1 \times 2} & \mathbf{0}_{1 \times 2} \\
\mathbf{0}_{3 \times 2} & \mathbf{P}_{\perp}
\end{array}\right]^{T} \quad \mathbf{S}_{\perp}\left[\begin{array}{cc}
\mathbf{P}_{\perp} & \mathbf{0}_{3 \times 2} \\
\mathbf{0}_{1 \times 2} & \mathbf{0}_{1 \times 2} \\
\mathbf{0}_{3 \times 2} & \mathbf{P}_{\perp}
\end{array}\right]\right\rangle, \\
& \mathbb{U}_{T H}=\left\langle\mathbb{P}_{T H}^{T} \mathbf{C}_{T}^{-1} \mathbb{P}_{T H}\right\rangle=\left\langle\frac{1}{J_{o}^{2}}\left[\begin{array}{cc}
\mathbf{P}_{T} & \mathbf{0}_{2 \times 1} \\
\mathbf{0}_{2 \times 1} & \mathbf{P}_{T}
\end{array}\right]^{T} \mathbf{S}_{T}\left[\begin{array}{cc}
\mathbf{P}_{T} & \mathbf{0}_{2 \times 1} \\
\mathbf{0}_{2 \times 1} & \mathbf{P}_{T}
\end{array}\right]\right\rangle
\end{aligned}
$$

where

$$
\mathbf{S}_{\perp}=\operatorname{diag} \cdot\left\{\mathbf{T}_{\varepsilon}^{T}, 0, \mathbf{T}_{\varepsilon}^{T}\right\} \cdot \tilde{\mathbf{C}}_{\perp}^{-1} \cdot \operatorname{diag} \cdot\left\{\mathbf{T}_{\varepsilon}, 0, \mathbf{T}_{\varepsilon}\right\}, \mathbf{S}_{T}=\operatorname{diag} \cdot\left\{\mathbf{T}_{\gamma}^{T}, \mathbf{T}_{\gamma}^{T}\right\} \cdot \mathbf{C}_{T}^{-1} \cdot \operatorname{diag} \cdot\left\{\mathbf{T}_{\gamma}, \mathbf{T}_{\gamma}\right\}
$$

Provided that the above flexibility matrices are still positive definite, it has been shown the element is still robust [15]. The following approximations are adopted for higher computational efficiency :

$$
J_{o} \simeq \bar{J}_{o}=\left.J_{o}\right|_{\xi=\eta=0}, \mathbf{S}_{\perp} \simeq \overline{\mathbf{S}}_{\perp}=\left.\mathbf{S}_{\perp}\right|_{\xi=\eta=0}, \mathbf{S}_{T} \simeq \overline{\mathbf{S}}_{T}=\left.\mathbf{S}_{T}\right|_{\xi=\eta=0}
$$

with which

$$
\begin{aligned}
& \mathbb{H}_{\perp H}=\frac{2}{\bar{J}_{o}} \int_{-1}^{+1+1} \int_{-1}^{+1}\left[\begin{array}{cc}
\mathbf{P}_{\perp} & \mathbf{0}_{3 \times 2} \\
\mathbf{0}_{1 \times 2} & \mathbf{0}_{1 \times 2} \\
\mathbf{0}_{3 \times 2} & \mathbf{P}_{\perp}
\end{array}\right]^{T} \overline{\mathbf{S}}_{\perp}\left[\begin{array}{cc}
\mathbf{P}_{\perp} & \mathbf{0}_{3 \times 2} \\
\mathbf{0}_{1 \times 2} & \mathbf{0}_{1 \times 2} \\
\mathbf{0}_{3 \times 2} & \mathbf{P}_{\perp}
\end{array}\right] d \xi d \eta=\frac{32}{15 \bar{J}_{o}} \operatorname{diag} .\left\{\bar{S}_{\perp 11}, \bar{S}_{\perp 22}, \bar{S}_{\perp 55}, \bar{S}_{\perp 66}\right\}, \\
& \mathbb{U}_{T H}=\frac{2}{\bar{J}_{o}} \int_{-1}^{+1+1} \int_{-1}^{+1}\left[\begin{array}{cc}
\mathbf{P}_{T} & \mathbf{0}_{2 \times 1} \\
\mathbf{0}_{2 \times 1} & \mathbf{P}_{T}
\end{array}\right]^{T} \overline{\mathbf{S}}_{T}\left[\begin{array}{cc}
\mathbf{P}_{T} & \mathbf{0}_{2 \times 1} \\
\mathbf{0}_{2 \times 1} & \mathbf{P}_{T}
\end{array}\right] d \xi d \eta=\frac{32}{15 \bar{J}_{o}}\left[\begin{array}{ll}
\bar{S}_{T 11}+\bar{S}_{T 22} & \bar{S}_{T 13}+\bar{S}_{T 24} \\
\bar{S}_{T 31}+\bar{S}_{T 42} & \bar{S}_{T 33}+\bar{S}_{T 44}
\end{array}\right]
\end{aligned}
$$

where $\left[\bar{S}_{\perp i j}\right]=\overline{\mathbf{S}}_{\perp}$ and $\left[\bar{S}_{T i j}\right]=\overline{\mathbf{S}}_{T}$. Continuing from (31), the stabilized element stiffness matrix would be :

$$
\begin{aligned}
\tilde{\mathbb{K}}_{S}=\tilde{\mathbb{K}}_{L}+ & \frac{15 J_{o}}{32}\left(\frac{1}{\bar{S}_{\perp 11}} \mathbb{G}_{m 1}^{T} \mathbb{G}_{m 1}+\frac{1}{\bar{S}_{\perp 22}} \mathbb{G}_{m 2}^{T} \mathbb{G}_{m 2}+\frac{1}{\bar{S}_{\perp 55}} \mathbb{G}_{b 1}^{T} \mathbb{G}_{b 1}+\frac{1}{\bar{S}_{\perp 66}} \mathbb{G}_{b 2}^{T} \mathbb{G}_{b 2}\right) \\
& +\frac{15 J_{o}}{32}\left(\left[\begin{array}{c}
\mathbb{G}_{o} \\
\mathbb{G}_{n}
\end{array}\right]^{T}\left[\begin{array}{ll}
\bar{S}_{T 11}+\bar{S}_{T 22} & \bar{S}_{T 13}+\bar{S}_{T 24} \\
\bar{S}_{T 31}+\bar{S}_{T 42} & \bar{S}_{T 33}+\bar{S}_{T 44}
\end{array}\right]^{-1}\left[\begin{array}{c}
\mathbb{G}_{o} \\
\mathbb{G}_{n}
\end{array}\right]\right)
\end{aligned}
$$

The expensive $3 \times 3$ integration scheme is not required in computing element stiffness matrix. 


\section{NUMERICAL EXAMPLES}

In this section, a number of benchmark problems will be studied by the following eighteen-node solid-shell elements :

- PS_R — the reduced integrated (by $2 \times 2$ rule) plane stress element $\mathbb{K}^{P S}$, see (14).

- PS_zeta - the reduced integrated (by $2 \times 2$ rule) plane stress element that accounts for the quadratic $\zeta$-terms in the inplane strains and the all the $\zeta$-terms in the Jacobian determinant, third order quadrature is employed for the thickness integration.

- PS_F — the fully integrated (by $3 \times 3$ rule) plane stress element $\mathbb{K}^{P S}$, see (14).

- present — the present stabilized element that employed the modified generalized laminate

stiffness matrix, see (40).

Moreover, the predictions of the hybrid-strain eighteen-node element CSHEL9 for composite plates and shells will be quoted as far as possible [13].

\subsection{Patch Tests}

All the four elements pass the constant moment, constant membrane stress and constant transverse shear stress patch tests stipulated by MacNeal \& Harder [17] for Mindlin plate elements.

\subsection{Clamped Circular Plate}

Figure 3 shows a quadrant of a clamped circular plate modelled by three and twelve elements. The plate is loaded transversely by either a central point force or a uniformly distributed force. To test the elements against shear locking, two radius $R$ to thickness $h$ ratios are considered. Table 3 lists the central deflections which have been normalized by the thin plate solutions. It can be seen that only PS_F suffers from shear locking in this problem. 
Table 2 : Normalized central deflections for centrally loaded clamped circular plate (Figure 3 )

\begin{tabular}{|c|c|c|c|c|c|c|c|c|}
\hline & \multicolumn{4}{|c|}{ central point force } & \multicolumn{3}{c|}{ uniformly distributed force } \\
\cline { 2 - 8 } & \multicolumn{2}{|c|}{3 elements } & \multicolumn{2}{c|}{12 elements } & \multicolumn{2}{c|}{3 elements } & \multicolumn{2}{c|}{12 elements } \\
\hline$R / h$ & 10 & 1000 & 10 & 1000 & 10 & 1000 & 10 & 1000 \\
\hline PS_R* & 1.142 & 1.047 & 1.127 & 1.006 & 1.032 & 0.995 & 1.037 & 0.999 \\
PS_F & 0.935 & 0.056 & 1.067 & 0.636 & 0.941 & 0.628 & 1.027 & 0.656 \\
present & 1.100 & 1.025 & 1.092 & 1.001 & 1.043 & 1.004 & 1.038 & 1.001 \\
\hline
\end{tabular}

* PS zeta is identical to PS R under flat plate geometry

\section{3 $90^{\circ}$ Pre-twisted Cantilever}

Figure 4 depicts the twisted beam which is doubly-curved and modelled by only six elements. It constitutes a good test for membrane locking [18] and examining the effect of including the higher order $\zeta$-terms in PS_zeta. At the clamped end, all nodal d.o.f.s are restrained. In-plane and out-ofplane end forces are applied to the free end. To test whether the elements exhibit locking, two thickness $h$ 's are considered. The normalized end deflections with respect to the reference solutions [18] are tabulated in Table 3. Only the fully integrated element exhibits locking in this problem. Differences between the predictions of PS_R and PS_zeta are limited to $0.2 \%$.

Table 3 : Normalized end deflections for the twisted beam problem (Figure 4)

\begin{tabular}{|c|c|c|c|c|}
\hline & \multicolumn{2}{|c|}{ in-plane loading } & \multicolumn{2}{c|}{ out-of-plane loading } \\
\cline { 2 - 5 } & $h=0.32$ & $h=0.0032$ & $h=0.32$ & $h=0.0032$ \\
\hline PS_R & 1.006 & 1.015 & 1.003 & 1.004 \\
PS_zeta & 1.006 & 1.016 & 1.002 & 1.006 \\
PS_F & 0.982 & 0.007 & 0.984 & 0.007 \\
present & 1.003 & 1.010 & 1.001 & 1.009 \\
\hline
\end{tabular}

\subsection{Hemispherical Shell with a $18^{\circ}$ Cutout at the Pole}

Figure 5 shows a quadrant of a hemispherical shell with a $18^{\mathrm{O}}$ circular cutout at its pole. It can be seen that this problem is also doubly curved. Two pairs of diametrical forces act along the $X$ - and $Y$-axes. As a result of symmetry, one quarter of the shell is modelled. Each element in the mesh subtends equal incremental values of $\phi$ and $\theta$. Deflections along the Y-axis at point $\mathrm{A}$ are normalized by the reference solution [17] and listed in Table 4. Accuracy of PS_R and the present element are extremely close. Without being treated for membrane and shear lockings, PS_F is very poor in accuracy. Differences between the predictions of PS_R and PS_zeta are less than 0.05\%. 
Table 4 : Normalized deflections for the hemispherical shell problem (Figure 5)

\begin{tabular}{|c|c|c|c|c|c|}
\hline no. of elements & $2 \times 2$ & $3 \times 3$ & $4 \times 4$ & $6 \times 6$ & $7 \times 7$ \\
\hline PS_R & 1.096 & 1.027 & 1.012 & 1.002 & 1.000 \\
PS_zeta & 1.096 & 1.027 & 1.012 & 1.002 & 1.000 \\
PS_F & 0.002 & 0.007 & 0.022 & 0.100 & 0.170 \\
present & 1.038 & 1.010 & 1.005 & 1.000 & 0.999 \\
\hline
\end{tabular}

\subsection{Pinched Cylindrical Shell}

Figure 6 shows a cylindrical shell mounted over two rigid end diaphragms. At the midspane, the shell is loaded by a pair of diametrically opposite point forces. Owing to symmetry, one octant of the shell is analyzed. The deflections under the point force are computed and normalized by the reference solution [18]. Table 5 lists the results. The present element is most accurate. The differences between the predictions of PS_R and PS_zeta are within $0.1 \%$.

Table 5 : Normalized deflections for pinched cylindrical shell

\begin{tabular}{|c|c|c|c|c|c|}
\hline no. of elements & $2 \times 2$ & $4 \times 4$ & $8 \times 8$ & $12 \times 12$ & $16 \times 16$ \\
\hline PS_R & 2.461 & 1.075 & 1.016 & 1.019 & 1.020 \\
PS_zeta & 2.460 & 1.074 & 0.016 & 1.018 & 1.020 \\
PS_F & 0.046 & 0.156 & 0.560 & 0.805 & 0.903 \\
present & 0.763 & 0.949 & 0.998 & 1.006 & 1.009 \\
\hline
\end{tabular}

\subsection{Clamped Cross-Ply Square Plate}

This problem was considered by Haas \& Lee [13]. A fully clamped square plate under an uniform distributed load $q$ is examined. The material properties are $E_{L}=40 E_{T}, G_{L T}=0.5 E_{T}, v_{L T}=v_{T T}=0.25$. The $-45^{\circ} / 45^{\circ}$ lay-up is considered for a range of side length $L$ to thickness $h$ ratios. A uniform $8 \times 8$ mesh is used to model the entire plate. The non-dimensional central deflections $\bar{w}=1000 w E_{T} h^{3} / L^{4} q$ are given in Table 6 . Judging from four significant figures, PS_R and the present element yield identical results which get closer to that of CSHEL9 when $L / h$ increases.

Table 6 : Non-dimensional central deflections $1000 w E_{T} h^{3} / L^{4} q$ for clamped cross-ply square plates

\begin{tabular}{|c|c|c|c|c|c|c|}
\hline$L / h$ & 25 & 50 & 100 & 200 & 500 & 1000 \\
\hline PS_R* & 3.517 & 3.123 & 3.014 & 2.986 & 2.977 & 2.976 \\
\hline present & 3.517 & 3.123 & 3.014 & 2.986 & 2.977 & 2.976 \\
\hline CSHEL9 [13] & 3.298 & 3.034 & 3.000 & 2.983 & 2.978 & 2.977 \\
\hline
\end{tabular}

* PS_zeta is identical to PS_R under flat plate geometry 


\subsection{Clamped Cylinder under Internal Pressure}

The clamped cylindrical shell in Figure 7 is subjected to internal pressure $P$ and solved for the maximum radial displacement. The material properties, pressure and geometry dimensions are $E_{L}=$ $7.5 \times 10^{6} \mathrm{psi}, E_{T}=2.0 \times 10^{6} \mathrm{psi}, G_{T T}=1.25 \times 10^{6} \mathrm{psi}, G_{L T}=0.625 \times 10^{6} \mathrm{psi}, \nu_{L T}=\mathrm{v}_{T T}=0.25, \mathrm{P}=(6.41 / \pi)$ psi, $R=20$ in and $L=20$ in. Two $R / h$ ratios are considered. Since the laminations are symmetric, only one octant of the cylinder is analyzed and a $8 \times 8$ uniform mesh is employed. The present predictions are close to that of CSHEL9. PS_R and PS_zeta differs by not more than $0.2 \%$.

Table 7 : Maximum radial displacement a clamped $90^{\circ}$ cylindrical shell, see Figure

\begin{tabular}{|c|c|c|c|c|}
\hline \multicolumn{2}{|c|}{ lamination } & $0^{\circ}$ & $\left(+45^{\circ} /-45^{\circ}\right)_{\mathrm{S}}$ & $\left(0^{\circ} / 90^{\circ}\right)_{\mathrm{S}}$ \\
\hline$R / h=20$ & PS_R & $0.0003636^{*}$ & 0.0002357 & 0.0001739 \\
& PS_zeta & $0.0003636^{*}$ & 0.0002361 & 0.0001739 \\
& present & $0.0003685^{*}$ & 0.0002399 & 0.0001795 \\
& CSHEL9 [13] & $0.0003781^{*}$ & 0.0002402 & 0.0001783 \\
\hline \multirow{4}{*}{$R / h=100$} & PS_R & 0.002035 & 0.001064 & 0.0008375 \\
& PS_zeta & 0.002035 & 0.001064 & 0.0008375 \\
& present & 0.002040 & 0.001068 & 0.0008414 \\
& CSHEL9 [13] & 0.002044 & 0.001068 & 0.0008422 \\
\hline
\end{tabular}

* the solution bases on a two-dimensional thin shell theory is 0.0003666 [13].

\subsection{Clamped $90^{\circ}$ Cylindrical Shell under Internal Pressure}

Figure 8 shows a fully clamped $90^{\circ}$ section of a cylinder. The material properties, pressure, radius and longitudinal length are the same as the last example. Several $R$ to $h$ ratios are considered and the results for the central radial deflection are presented in Table 8. Once again, the present predictions are close to that of CSHEL9. In this example, PS_R and PS_zeta differs by not more than $0.05 \%$.

Table 8 : Radial displacement at the center of clamped $90^{\circ}$ cylindrical shell, see Figure 6

\begin{tabular}{|c|c|c|c|c|c|}
\hline \multicolumn{2}{|c|}{ lamination } & $0^{\circ}$ & $-45^{\circ} /+45^{\circ}$ & $\left(+45^{\circ} / 45^{\circ}\right)_{\mathrm{S}}$ & $\left(0^{\circ} / 90^{\circ}\right)_{\mathrm{S}}$ \\
\hline$R / h=20$ & PS_R & 0.0004260 & 0.0002848 & 0.0002837 & 0.0002247 \\
with $10 \times 10$ & PS_zeta & 0.0004260 & 0.0002847 & 0.0002837 & 0.0002248 \\
elements & present & 0.0004312 & 0.0002899 & 0.0002889 & 0.0002275 \\
& CSHEL9[13] & 0.0004367 & 0.0002916 & 0.0002909 & 0.0002300 \\
\hline$R / h=100$ & PS_R & 0.001867 & 0.0008888 & 0.0008918 & 0.0007384 \\
with $12 \times 12$ & PS_zeta & 0.001867 & 0.0008888 & 0.0008918 & 0.0007383 \\
elements & present & 0.001870 & 0.0008908 & 0.0008939 & 0.0007396 \\
& CSHEL9[13] & 0.001877 & 0.0008936 & 0.0008965 & 0.0007424 \\
$R / h=300$ & PS_R & 0.006171 & 0.003331 & 0.003296 & 0.002603 \\
with $12 \times 12$ & PS_zeta & 0.006171 & 0.003330 & 0.003296 & 0.002603 \\
elements & present & 0.006179 & 0.003335 & 0.003300 & 0.002606 \\
& CSHEL9[13] & 0.006208 & 0.003351 & 0.003314 & 0.002621 \\
\hline
\end{tabular}




\section{CLOSURE}

By assuming the thickness stress being independent of $\zeta$, realizing that the thickness strain for solid-shell elements with two layers of node can only represent its average value and neglecting the second order $\zeta$-terms in the inplane strain as well as all the $\zeta$-terms in the Jacobian determinant, this paper presents a modified generalized laminate stiffness matrix that can resolve thickness locking without resorting to any advanced finite element technique. A modified potential energy functional is devised that calculates the strain energy from the membrane, bending, average thickness and transverse shear strains via the modified generalized laminate stiffness matrix. While the assumption on the thickness stress can lead to a better satisfaction of the traction reciprocity at the interlaminar interfaces, it is noted by comparing PS_R and PS_zeta that the neglected $\zeta$-terms in the inplane strain and the Jacobian determinant do not lead to any marked difference in the element predictions.

A modified Hellinger-Reisssner functional is formed by relaxing the constitutive relation in the modified potential energy functional. With the former functional, the previous hybrid-stabilized eighteen-node solid-shell element formulated for homogenous materials using plane stress enforcement [7] is successfully extended to laminate plate/shell analysis. Compared to the stabilized element of Lee \& Haas [13], the present element is more efficient due to the energy orthogonality of the lower and higher order stress modes and the explicit nature of the higher order leverage matrices.

By discarding the thickness stress/strain and adopting the plane stress constitutive relation, the modified Hellinger-Reissner functional given in Appendix can be employed for formulating degenerated-shell elements for laminated plate and shell analyses.

Acknowledgment - The work described in this paper was supported by a grant from the Research Grant Council of Hong Kong SAR (Project Number : HKU 7111/99E). 


\section{APPENDIX}

For degenerated-shells, the plane stress condition is assumed and $\boldsymbol{\gamma}_{n}=\mathbf{0}$. The corresponding modified potential energy functional and the modified Hellinger-Reissner functional are respectively :

$$
\Pi_{m P}^{e}=\frac{1}{2}\left\langle\left\{\begin{array}{l}
\boldsymbol{\epsilon}_{m} \\
\boldsymbol{\epsilon}_{b}
\end{array}\right\}^{T} \mathbf{C}_{m b}\left\{\begin{array}{l}
\boldsymbol{\epsilon}_{m} \\
\boldsymbol{\epsilon}_{b}
\end{array}\right\}+\boldsymbol{\gamma}_{o}^{T} \mathbf{C}_{t o} \boldsymbol{\gamma}_{o}\right\rangle-P^{e}
$$

and

$$
\Pi^{e}=\left\langle-\frac{1}{2}\left\{\begin{array}{l}
\mathbf{N} \\
\mathbf{M}
\end{array}\right\}^{T} \mathbf{C}_{m b}^{-1}\left\{\begin{array}{l}
\mathbf{N} \\
\mathbf{M}
\end{array}\right\}+\left\{\begin{array}{l}
\mathbf{N} \\
\mathbf{M}
\end{array}\right\}^{T}\left\{\begin{array}{l}
\boldsymbol{\epsilon}_{m} \\
\boldsymbol{\epsilon}_{b}
\end{array}\right\}-\frac{1}{2} \mathbf{Q}_{o}^{T} \mathbf{C}_{t o}^{-1} \mathbf{Q}_{o}+\mathbf{Q}_{o}^{T} \boldsymbol{\gamma}_{o}\right\rangle-P^{e}
$$

where

$$
\mathbf{C}_{m b}=\frac{1}{2} \int_{-1}^{+1}\left[\begin{array}{cc}
\mathbf{S}_{=}^{-1} & \zeta \mathbf{S}_{=}^{-1} \\
\zeta \mathbf{S}_{=}^{-1} & \zeta^{2} \mathbf{S}_{=}^{-1}
\end{array}\right] d \zeta \quad \text { and } \quad \mathbf{C}_{t o}=\frac{1}{2} \int_{-1}^{+1} \mathbf{C}_{t} d \zeta
$$

With the latter functional, hybrid-stress degenerated-shell elements for laminated plate/shell analyses can be formulated. 


\section{REFERENCES}

[1] M.F.Ausserer and S.W.Lee, "An eighteen-node solid element for thin shell analysis", Inter.J. Numer.Methods Engrg., 26, 1345-1364 (1988).

[2] Y.H.Kim and S.W.Lee, "A solid element formulation for large deflection analysis of composite shell structures", Computers \& Structures, 30, 269-274 (1988).

[3] K.Y.Sze and A.Ghali, "An hexahedral element for plates, shells and beams by selective scaling”, Inter.J.Numer.Methods Engrg., 36, 1519-1540 (1993).

[4] H.C.Park, C.Cho and S.W.Lee, "An efficient assumed strain element model with six dof per node for geometrically nonlinear shells", Inter.J.Numer.Methods Engrg., 38, 4101-4122 (1995).

[5] D.Roehl and E.Ramm, "Large elasto-plastic finite element analysis of solids and shells with the enhanced assumed strain concept", Inter.J.Solids \& Structures., 33, 3215-3237 (1996).

[6] P.Betsch, F.Gruttmann and E.Stein, "A 4-node finite shell element for the implementation of general hyperelastic 3D-elasticity at finite strains", Comput.Methods Appl.Mech.Engrg., 130, 57-79 (1996).

[7] K.Y.Sze, S.Yi and M.H.Tay, "An explicit hybrid-stabilized eighteen-node solid element for thin shell analysis", Inter.J.Numer.Methods Methods Engrg., 40, 1839-1856 (1997).

[8] R.Hauptmann and K.Schweizerhof, "A systematic development of solid-shell element formulations for linear and non-linear analysis employing only displacement degrees of freedom", Inter.J. Numer.Methods Engrg., 42, 49-69 (1988).

[9] K.Y.Sze, L.-Q.Yao and S.Yi, "A hybrid-stress ANS solid-shell element and its generalization to smart structure modeling", Inter.J.Numer.Methods Engrg., 48, 545-582 (2000).

[10] R.H.MacNeal, "A theorem regarding the locking of tappered four-noded membrane elements", Inter.J.Numer.Methods Engrg., 24, 1793-1799 (1987).

[11] K.Y.Sze, "On immunizing five-beta hybrid stress elements from 'trapezoidal locking' in practical analyses”, Inter.J. Numerical Methods Engrg., 47, 907-920 (2000).

[12] T.H.H.Pian, "Finite elements based on consistently assumed stresses and displacements", Finite Elements in Analysis \& Design, 1, 131-140 (1985).

[13] D.J.Haas and S.W.Lee, "A nine-node assumed-strain finite element for composite plates and shells", Computers and structures, 26, 445-452 (1987).

[14] C.Sansour and F.G.Kollmann, "Families of 4-node and 9-node finite elements for a finite deformation theory. An assessment of hybrid stress, hybrid strain and enhanced strain elements", Computional Mechanics, 24, 435-447 (2000)

[15] K.Y.Sze, "Admissible matrix formulation : from orthogonal approach to explicit hybridstabilization", Finite Elements in Analysis \& Design, 24, 1-30 (1996).

[16] O.C.Zienkiewicz, R.L.Taylor, The Finite Element Method, Vol.1 - Basic Formulation and Linear Problems, $4^{\text {th }}$ Edn., McGraw-Hill, London, 1989

[17] R.H.MacNeal and R.L.Harder, "A proposed standard set of problems to test finite element accuracy", Finite Elements in Analysis \& Design, 1, 3-20 (1985).

[18] T.Belytschko, B.K.Wong and H.Stolarski, "Assumed strain stabilization procedure for the 9node Lagrange shell element”, Inter.J.Numer.Methods.Engrg., 28, 385-414 (1989). 


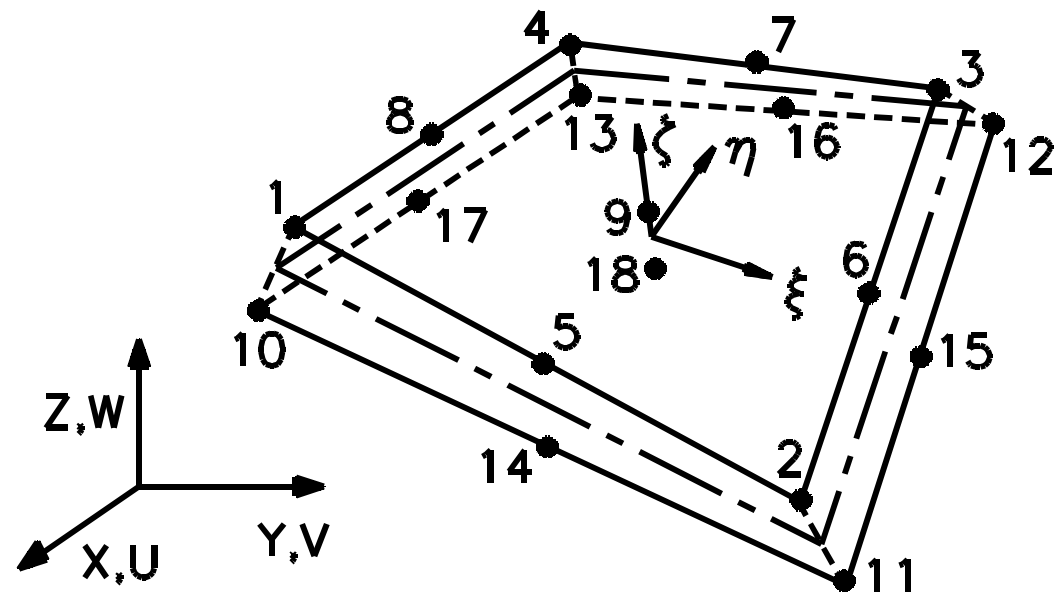

Figure 1 : An eighteen-node solid-shell element

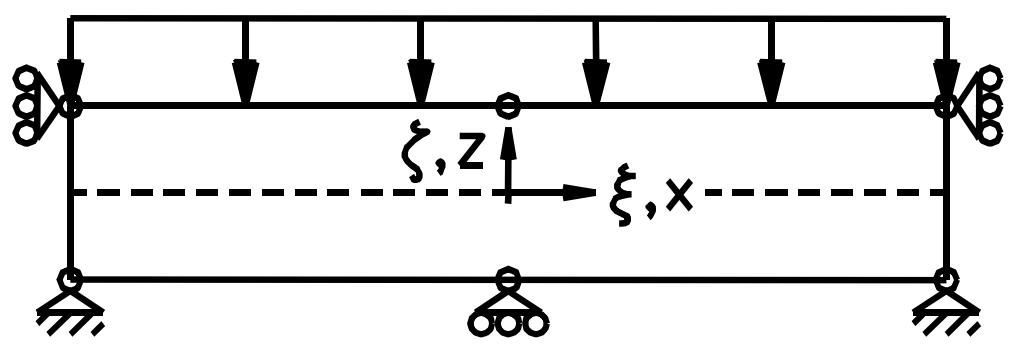

(a)

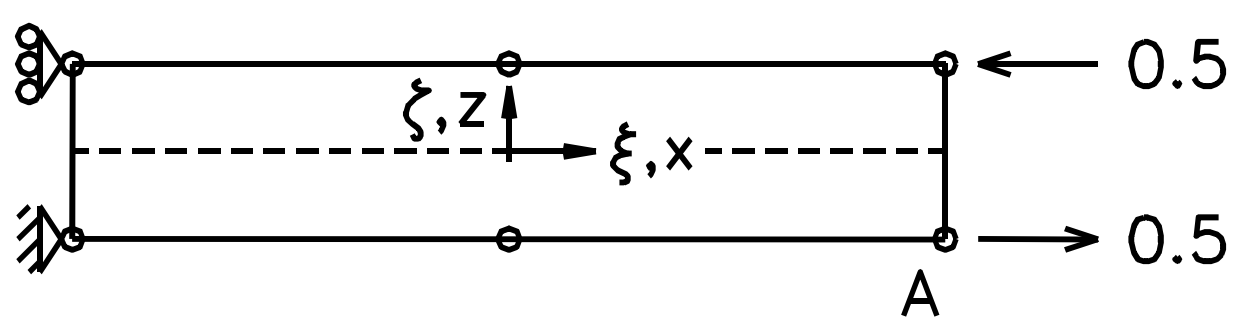

(b)

Figure 2: A 10×2 two-ply six-node element subjected to (a) uniform compressive stress and (b) end moment 

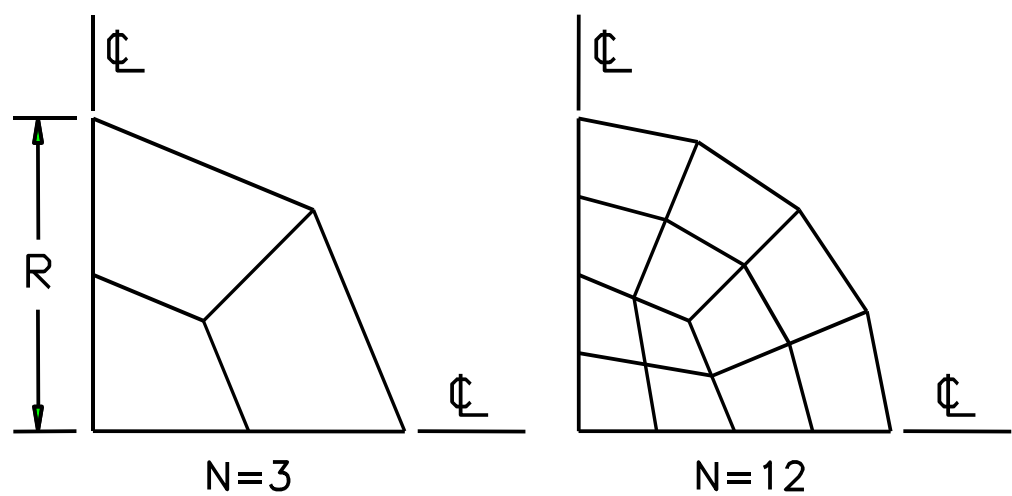

Figure 3: Quadrant of a circular plate modelled by 3 and 12 elements

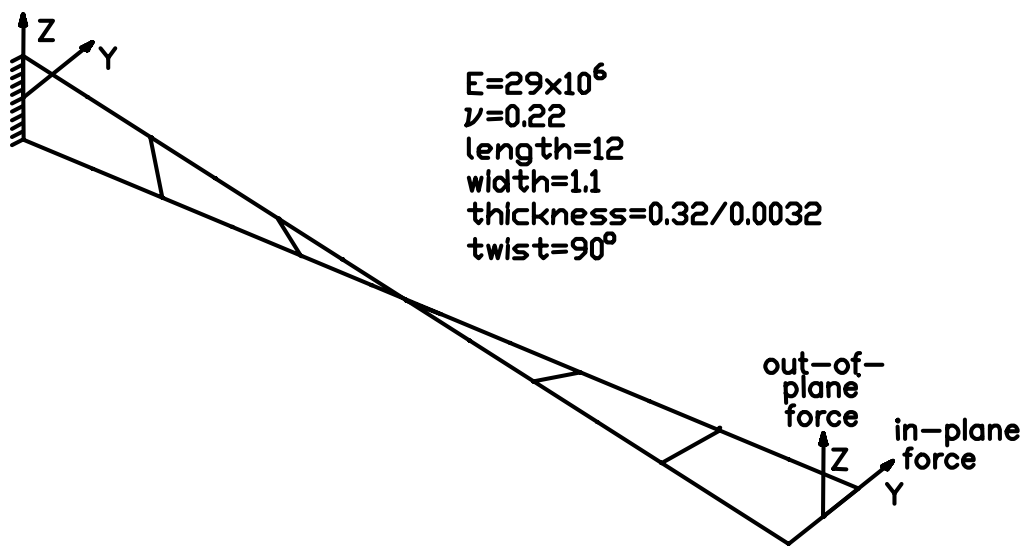

Figure 4 : A $90^{\circ}$ twisted beam modelled by a $1 \times 6$ elements.

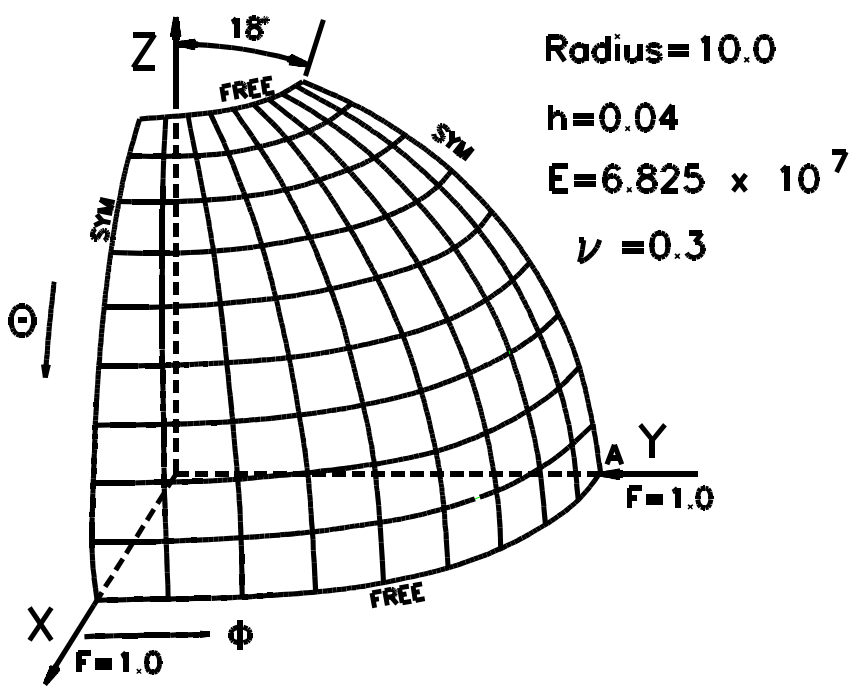

Figure 5 : A hemispherical shell with $18^{\circ}$ cutout modelled by $9 \times 9$ elements 


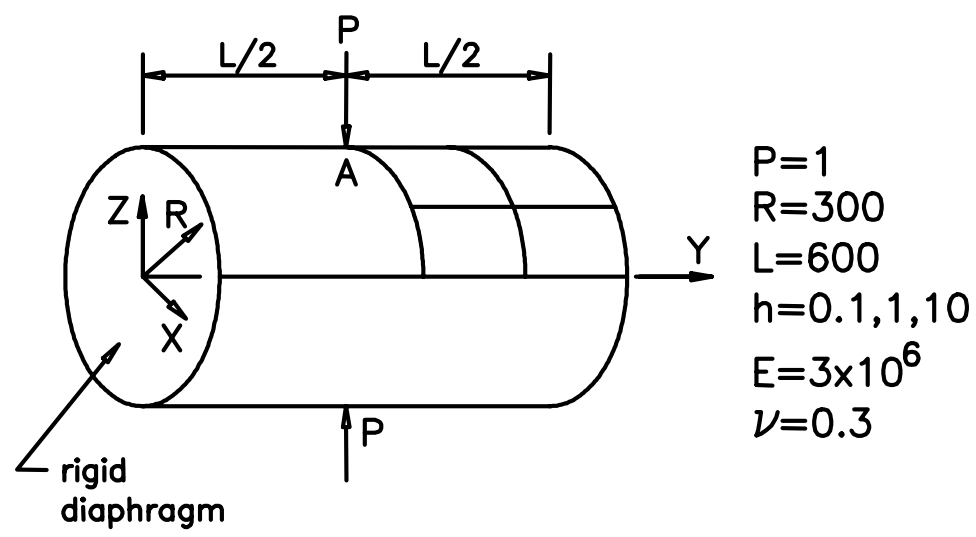

Figure 6 : The pinched cylindrical shell problem modelled by $2 \times 2$ elements.

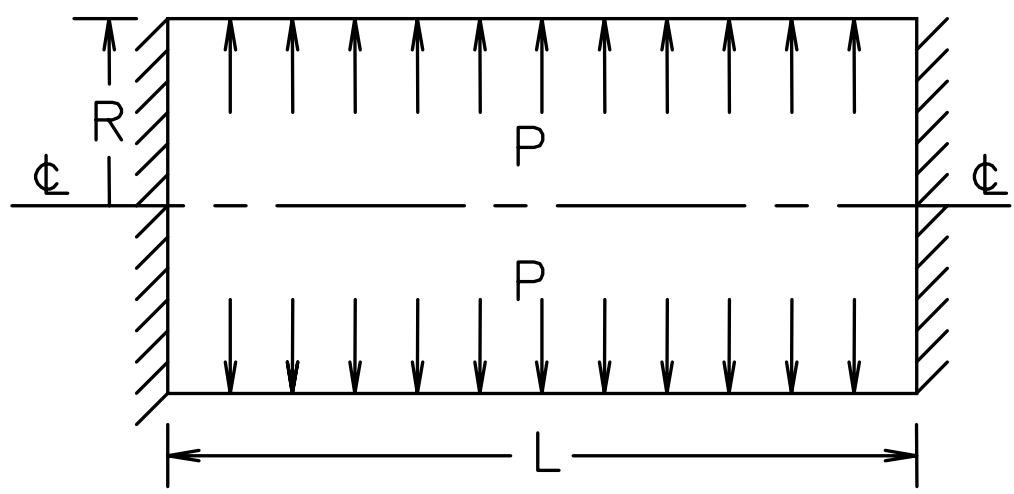

Figure 7 : Clamped cylindrical shell subjected to internal internal pressure $\mathrm{P}$.

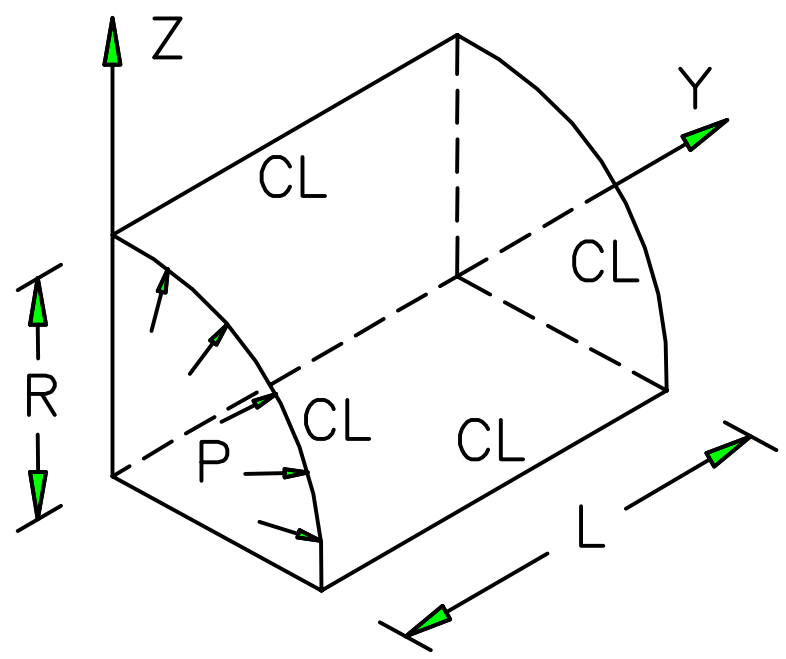

Figure 8 : Clamped $90^{\circ}$ cylindrical shell subjected to internal internal pressure $\mathrm{P}$. 